\title{
Simulation of Blood Flow past a Distal Arteriovenous-Graft Anastomosis at Low Reynolds Numbers
}

\author{
Zengding Bai ${ }^{\mathrm{a}}$, Luoding $\mathrm{Zhu}^{\mathrm{a}, *}$ \\ ${ }^{a}$ Department of Mathematical Sciences, Indiana University-Purdue University \\ Indianapolis,402 North Blackford Street, Indianapolis, IN 46202, USA
}

\begin{abstract}
Patients with end-stage renal disease (ESRD) are usually treated by hemodialysis (HD) while waiting for kidney transplant. A common device for vascular access is arteriovenous graft (AVG). However, AVG failure induced by thrombosis has been plaguing dialysis practice for decades. Current studies indicate that the thrombosis is caused by intimal hyperplasia (IH) which is triggered by the abnormal flows and forces (e.g. wall shear stress) in the vein after AVG implant. Due to high level of complexity, in almost all of the existing works of modeling and simulation of the blood-flow vessel-AVG system the graft and blood-vessel are assumed to be rigid and immobile. Very recently we have found that the compliance of graft and vein can reduce flow disturbances and lower wall shear stress (WSS) (Z Bai and L Zhu, Computers \& Fluids 181, pp. 403$415,2019)$. In this paper, we apply the compliant model to investigate possible effects of several dimensionless parameters (AVG graft-vein diameter ratio $R_{g v}$, AVG attaching angle $\theta$, flow Reynolds numbers $R e$, and native vein speed $V_{v}$ ) on the flow and force fields near the distal AVG anastomosis at low Reynolds numbers (up to several hundreds). Our computational results indicate that the influences of the parameters $R_{g v}, \theta$, and Re lie largely on the graft and the influence of $V_{v}$ lies largely on the vein. In any case, the wall shear stress (WSS),
\end{abstract}

\footnotetext{
* Corresponding author

Email address: luozhu@iupui.edu (Luoding Zhu)
} 
wall shear stress gradient (WSSG), and wall normal stress gradient (WNSG) and their averaged values on the graft are significantly greater than those on the vein.

Keywords: End-stage renal disease, Dialysis, Arteriovenous graft, Intimal hyperplasia, Blood flow, Biofluid dynamics, Fluid-structure interaction, Immersed boundary, lattice Boltzmann, Wall shear stress

\section{Introduction}

Kidney failure, also called end-stage renal disease (ESRD), is the last and most severe stage of chronic kidney disease. When kidneys fail, their renal excretory and regulatory functions are no longer enough for people to survive without hemodialysis (HD) or a kidney transplant. Hemodialysis is a process of purifying the blood of a patient by an artificial kidney to remove waste products. Even though the kidney transplant seems able to provide a complete solution, the limited supply of donor kidney makes it possible for only a small portion of ESRD patients. The majority of the patients have to rely on dialysis to maintain their lives.

In dialysis, the artificial kidney needs to access blood from the patient's artery, has blood circulated and cleaned through the machine, and finally returns the blood to the patient's vein. One major type of vascular access is arteriovenous graft (AVG). An AVG is a piece of artificial tubing, generally made out of polytetrafluoroethylene (PTFE), which is attached on one end to an artery, and on the other end to a vein. The tube is permanently implanted usually in the patient's forearm and is punctured by two needles to allow the circulation process to occur in dialysis machine.

A major problem challenging dialysis for decades is the blockage of the artificially created shunt by thrombosis which is caused by occlusion of the outflow venous anastomosis and $\square$ draining vein $[1,2]$. Note that the alternative approach arteriovenous fistula (AVF) faces the similar problem (see Brahmbhatt et al. [3]). Pathologic study of the outflow graft-vein anastomoses of PTFE 
grafts with thrombosis discovered intimal hyperplasia (IH) at the site of venous anastomosis and in the proximal $[$ downstream vein [4]. Intimal hyperplasia $(\mathrm{IH})$, the thickening of the innermost layer of vessel wall, is formed by excessive migration and increase of vascular smooth muscle cells (VSMC) in the intima. Miller [5], Lemson [6], and Haruguchi [7] pointed out that intimal hyperplasia is the initial pathological event leading to shunt thrombosis.

Intimal hyperplasia has attracted extensive studies. The most significant initiating factors include the injury caused by AVG implantation and the disturbed blood flows from the AVG outlet, which are typically pulsatile and have high speed compared to the original native vein flows which are slow and quasisteady. See Cunnane et al. [8] for a recent review on hemodynamic factors contributing to the onset of intimal hyperplasia.

It has been shown that vascular endothelial cells (VEC) lining the vein's innermost wall are sensitive to the wall shear stress (WSS), wall normal stress (WNS), and their gradients $[9,10,11]$. Abnormal values of these biomechanical variables are thought to be the culprit of initialization and development of the intimal hyperplasia (IH). In fact, there are many such studies in current literatures. Dobrin (1989), Ojha (1990), Kraiss (1991), Cinat (1999), Mima (2012), Clowes (1983), Fillinger (1990), and Hofstra (1995) reported that IH was associated with the significantly altered (lowered or elevated) WSS near the graft-vein anastor osi $412,13,14,15,16,17,18,19]$. Thubrikar et al. [20] speculated that it was the dramatically increased WNS that led to the IH. Some researchers believed that the IH was due to the stimulation of the flow disturbances near the anas tomosis [21, 22, 23. 24]. Fulker et al.[25] investigated the flow disturbance and turbulence induced by the dialysis needles in a patient specific fistula by computational fluid dynanids. Grechy et al. [26] applied CFD and optimization to design a novel AVF configuration for the suppression of flow unsteadiness.

However, direct measurement of these mechanical quantities in laboratory (in vivo or in vitro) are difficult and such data are not currently available in literature. Instead, the in silico approach, i.e., the Computational Fluid Dynamics (CFD) has long been applied to gauge the flow and force fields of the 
flow-graft-ve:se. system $\square 27.28,29.30 .31 .32,33,34,35,36,37]$. See the paper by Ene-Iordache et al. [38] for a recent review on computational studies along this line. To the best of our knowledge, in almost all of the relevant studies including the above mentioned ones except McNally et al. [37], the blood vessel and AVG were assumed to be rigid and fixed. Thus the vessel/AVG radial contraction/dilation and axial compression/stretching were omitted. McNally et al.[37] used an elastic graft-vein model by commercial software ANSYS to study the efficacy of a novel device for reducing WSS on the vein and flow disturbance from the graft for three different flow rates.

However, genuine vein and AVG are flexible and may deform/move with the pulsatile flow: blood flowing through graft-vein is a typical fluid-structureinteraction problem and such interaction may alter the flow and force fields in conplicatt ranners [39, 40, 41]. Decorato et al. [42] and McGah et al. [43] found that the rigid assumption overestimated the averaged WSS for blood flow past an arteriovenous fistula (AVF). Very recently we [44] have introduced a compliant anastomosis model of arteriovenous graft (AVG) and investigated the effect of vein elasticity for given AVG configuration and flow condition. Our computational results have showed that the vein/graft flexibility can deaden flow disturbance and decrease wall shear stress (WSS) in the vein [44].

However, in dialysis the AVG configuration (graft-vein diameter ratio $R_{g v}$, attaching angle $\theta$ ), the flow Reynolds number $(R e)$, and the vein inlet speed $V_{v}$ are all variable. Here the Reynolds number is defined as $R e=\frac{U_{m} D}{\nu}$, where $U_{m}$ is twice of the mean speed of the graft inlet pulsatile flow, $D$ is the vein diameter, and $\nu$ is the fluid kinematic viscosity. These data differ substantially from patient to patient and from time to time for a specific patient. For example, the ratio $R_{g v}$ may be different because of different sizes of graft/vein selected for different patients and it may change over time because of the development of intimal hyperplasia. The flow Re may be different from sleeping to walking for a specific patient. Therefore, in this work, the compliant AVG anastomosis model in [44] is utilized to investigate possible effects of the AVG configuration $\left(R_{g v}\right.$ and $\theta$ ), the flow $R e$, and the vein inlet speed $V_{v}$ on the flow and force fields 
near the graft-vein anastomosis.

Because the parameters considered $\left(R_{g v}, \theta, R e\right.$, and $\left.V_{v}\right)$ are patient and time dependent, in the strict sense, patient specific data are not available (they change over time). Therefore, in this paper, we use several different values for each of these variables (including physiological values) and investigate their effects on flow and force fields near the distal anastomosis of the blood-flow graft-vein system. This is a unique features of our studies. To the best of our knowledge, such studies using compliant graft-vein model do not exist in literature.

The remaining article is listed as follows. Section 2 addresses the compliant anastomosis model. Section 3 gives the mathematical description. Section 4 outlines the numerical methods. Section 5 briefs verification and validation. Section 6 discusses major computational results. Section 7 summarizes and concludes the paper.

\section{Anastomosis Model}

Mathematical modeling of blood flow past the distal anastomosis of an arteriovenous-graft is challenging due to a variety of reasons. For example, the blood is an inhomogeneous multiphase non-Newtonian fluid; the vein and surrounding tissue are deformable porous living viscoelastic solid; the flow in the anastomosis could be deterministic or stochastic. As we always do in mathematical modeling of any real-world problem, we make assumptions to build an "ideal" model. To be complete and facilitate reading, the "ideal" compliant graft-vein anastomosis medel introduced in [44] is briefed in the following paragraph.

The blood is modelled by a homogeneous viscous Newtonian fluid and the blood flow by a laminar flow. The graft and vein are modelled by elastic circular tubes with different elasticity using Peskin's immersed bcundary framework [45]. Initially the vein is straight and the graft is curved; both are on the same plane and each is of constant diameter. The ambient tissue is modelled by elastic 
springs and viscous fluid. The inlets of the graft and vein and the vein outlets are fixed. The graft inlet velocity profile is taken from literature [46]. The vein inlet velocity is a fraction of the mean speed of the graft inlet speed. The flow at the vein outlet is friction free. We point out that many of these assumptions have already been used in literature. For exarple, [27, 33, 47, 48] treated blood flow as laminar flow of viscous Newtonian fluid; $[34,37]$ assumed that the graft and vein are on the same plane; $[49,48]$ used elastic springs to model surrounding tissue. Our model has the main advantage of incorporating the deformability of the graft and vein. As in the standard IB framework, the anastomosis model (a straight tube connected to a curved one in the middle) is situated in the center of a periodic rectangular box filled with stationary viscous Newtonian fluid. The standard rectilinear orthogonal Eulerian coordinate system is adopted: $\mathrm{x}$-axis points from left to right, $\mathrm{y}$-axis points from front to rear, and z-axis points from bottom to top. To facilitate understanding, a diagram of the anastomosis model is given in Fig. 1.

In dialysis, the dimensionless parameters $\left(R_{g v}, \theta, R e, V_{v}\right)$ are variables of patient and time. To investigate possible effects of these variables we pick several values for each parameter including the values used in dialysis. For example, attaching angle of 30 degree and ratio of 2:3 are commonly used in practice. The Re is high (hundreds to thousands) [50] for dialysis. But during non-dialysis period (the ratio between dialysis to non-dialysis is approximately 1:6), it is much lower, in particular, when the patient is at rest (e.g. sleep). Our LB-IB method is not stable for high Re flows. Therefore we use the Re range in 50 to 150 . Since the native blood flow at the vein inlet may be different for different patients, we choose to use four different speeds (nondimensionalized by the mean value of the blood flow at the graft inlet). See Table 1 for specific values of all parameters.

Notice that the flow at the graft inlet is pulsatile and there is another important dimensionless number, the Womersley number $\alpha$, defined as $\alpha=D \sqrt{\omega / \nu}$. Here $\omega$ is characteristic frequency, $D$ is characteristic length, and $\nu$ is fluid kinematic viscosity. Womersley number is related to Reynolds number by 
$\alpha^{2}=R e S t$, where $S t$ is the Strouhal number. In our simulations the $S t$ is approximately one, hence the value of Womersley number can be derived from Reynolds number: $\alpha=7.3,10.4,12.7$ for $R e=50,100,150$, respectively.

We point out that the goal of our work is to investigate possible effects of several parameters on the flow and force fields, rather than compute the specific flow and force fields of blood past an AVG anastomosis using fixed patient-specific data; therefore, all of the independent parameters and dependent variables (e.g. WSS) are kept dimensionless throughout the paper. Given an relevant patientspecific problem, one can convert the dimensionless data back to physical data via simi arity principle [51, 52].

\section{Mathematical Formulation}

The immersed boundary (IB) method describing the motions of both the blood and the graft-vein is given as follows.

$$
\begin{gathered}
\rho\left(\frac{\partial \mathbf{u}}{\partial t}+\mathbf{u} \cdot \nabla \mathbf{u}\right)=-\nabla p+\mu \Delta \mathbf{u}+\boldsymbol{b}_{f}(\boldsymbol{x}, t)+\int_{\Gamma} \boldsymbol{F}(\boldsymbol{\alpha}, t) \delta(\boldsymbol{x}-\boldsymbol{X}(\boldsymbol{\alpha}, t)) d \boldsymbol{\alpha} \\
\nabla \cdot \mathbf{u}=0 \\
\mathbf{F}(\boldsymbol{\alpha}, t)=-\frac{1}{2} \frac{\partial}{\partial \mathbf{X}}\left(\int_{\Gamma}\left(K_{s}\left(\left|\frac{\partial \mathbf{X}(\boldsymbol{\alpha}, \mathbf{t})}{\partial \boldsymbol{\alpha}}\right|-1\right)^{2}+K_{b}\left|\frac{\partial^{2} \mathbf{X}(\boldsymbol{\alpha}, t)}{\partial \boldsymbol{\alpha}^{2}}\right|^{2}\right) d \boldsymbol{\alpha}\right), \\
\frac{\partial \mathbf{X}}{\partial t}(\boldsymbol{\alpha}, t)=\mathbf{U}(\boldsymbol{\alpha}, t), \\
\boldsymbol{U}(\boldsymbol{\alpha}, t)=\int_{\Omega} \boldsymbol{u}(\boldsymbol{x}, t) \delta(\boldsymbol{x}-\boldsymbol{X}(\boldsymbol{\alpha}, t)) \mathrm{d} \boldsymbol{x} .
\end{gathered}
$$

Eqs. 1 and 2 are the incompressible Navier-Stokes equations governing motion of of viscous blood and structure (graft/vein). Here $\mathbf{u}, \rho$, and $p$ denotes velocity, mass density, and pressure of the liquid, respectively. The last term in 
Eq. 1 is the Eulerian force $\boldsymbol{f}_{i b}(\boldsymbol{x}, t)$ exerted by the graft and vein to the fluid ( $\boldsymbol{b}_{f}$ is other external force). Here $\boldsymbol{\alpha}$ and $\boldsymbol{X}$ are the Lagrangian and Eulerian coordinates of the graft and vein, respectively. The symbol $\delta$ is the Dirac delta function. The $\mathbf{F}$ is the Lagrangian force computed from the elastic potential energies (Eq. 3) of the graft and vein. The $K_{s}$ and $K_{b}$ are stretch/compression and flexure coefficients of elastic fibers constituting the graft and vein. They are related to Youngs' moduli and Poisson ratios of the graft and vein [53]. The velocity of the graft and vein $\boldsymbol{U}(\boldsymbol{\alpha}, t)$ is derived from the fulid velocity by Eq. 5 . Note that this equation dictates no-slip boundary condition on the fluid-solid interface.

\section{Numerical Method}

The immersed boundary formulation for the blood-flow graft-vein interac$\square$ tion, i.e., 届. 1 through Eq. 5, is a nonlinear system of PDE-ODE-integral equations. Analytical solutions of the system is virtually impossible except for extremely simplified cases. In general a computational approach has to be used. Finite difference, finite element, and finite volume methods have all been used for the discretization of the Navier-Stokes equations in the immersed boundary formulation. Here the lattice Boltzmann equations (the D3Q19 model) are used for this purpos [54 55] 56, 57,51,58,59,52]. The LB equations are relatively easier to implement, simpler to treat arbitrary rigid flow boundary, and more convenient to model additional flow physics $[59,52]$. The external force terms in the LB equations are discretized by the approach developed by Guo et al. [60]. Discretization of the remaining equations in the formulation uses the finite difference methods as in the original immersedboundary method [45]. For instances, the ihtegralls in Eqs. 1, 3, and 5 are discretized by the trapezoidal rule, the spatill derivatives in Eq. 3 are discretized by the centered difference, and the temporal derivative in Eq. 4 is discretized by the backward Euler method. The Dirac delta function is approximated by the cosine-version of the discretization in the immersed boundary framework [45]. 


\section{Verification and Validation}

The immersed boundary method has been very well tested and validated in a variety of situations (see [45] and references therein). The verification and validation of the lattice-Boltzmann based immersed boundary method (LB-IB) has been performed n previbus works [61, 62, 63, 64]. The implementation of the method used in this work for the blood-flow graft-vein system has been verified and validated by comparison with existing work and mesh refinement studies $[65,44]$. To demonstrate the computational results are convergent, we have performed simulations on a viscous fluid flowing in a flexible tube immersed in a three-dimensional domain [65] on a series of gradually refined fluid grids with grid-width successively halved (grid-nodes successively doubled from $10^{3}$ to $320^{3}$ ). The two ends of the tube are fixed and the fluid flows from left to right. Since the tube is flexible it deforms due to the flow-structure interaction. Because the interface separating fluids inside and outside of the tube is most critical to capture accurately, its shape and position serve as good gauge of Gonvergence. Fig. 2 plots the shape and position of the tube on six gradually refined grids. We can see that the convergence is attained on the grid of $80^{3}$.

Besides, the WSS on the graft and vein computed from our simulations are consistent with existing data in literature. The averaged dimensional WSS (converted from the LB units) in all the simulations is approximately between $0.06 \mathrm{~Pa}$ and 2.03 Pa. The relevant data in literature vary between less than 1 Pascals to hundreds of Pas als [27, 28, 31, 66, 33, 34, 35, 37]. In contrast, the WSS ranges from $0.1 \mathrm{~Pa}$ to $7 \mathrm{~Pa}$ for blood flow in normal blood vessels [67].

\section{Main computational results}

Numerous numerical simulations using various combinations of model parameters are designed and conducted. In this section, we report the major computational results and perform analyses through flow and force field visualization and qualitative/quantitative comparisons of the computational data. 
The focus is on the possible effects of three dimensionless parameters (graftvein ratio $R_{g v}$, graft-vein attaching angle $\theta$, and flow Reynolds number $R e$ ) and dimensionless vein inlet-speed $V_{v}$ on the flow and force fields near the distal anastomosis. A pulsatile velocity profile [46] is used at the graft inlet for all of the simulations (see Fig. 1). The pulsatile velocity profile is described and used in the previous work [44]. Our previous work using the compliant model [44] has demonstrated that the results at four instants of a typical pulsatile cycle are similar and do not show qualitative difference. Therefore only results at a typical instant (same for all results shown below) are reported here.

The simulation results are categorized into four groups. The model parameters adopted in each group are sum marized in Table 1 , where $s_{a}(t)$ represents the speed of the pulsatile flow on AVG inlet and its profile is depicted in [46]. Important graft-vein anastomosis configuration parameters include the diameter ratio of graft to vein $R_{g v}$ and the graft attaching angle $\theta$. In dialysis practice, these parameters are variable because the patient's vein selected for dialysis may have different size and shape, and the graft may comewith various size [68]. Besides, these parameters may change over time due to development of thrombosis and movements of the graft/vein and patient's forearm. Therefore, we investigate possible effects of these variables by using several values for each of these variables, including the values currently used in dialysis. For examples, attaching angle of 30 degree and ratio of 2:3 are commonly used in practice. The Reynolds number in dialysis is relatively ligh (1000-3000)[50]. But our LB-IB method is not stable for this range of Re. However, the Re may be much lower when the patient is at rest (e.g. sleep) during non-dialysis. And the ratio between dialysis to non-dialysis period is approximately 1:6. Therefore we use the Re range in 50 to 150 . Since the native blood flow at the inlet is patient and time dependent, we choose to use four different speeds (nondimensionalized by the mean value of the blood flow at the graft inlet).

Computed quantities such as WSS in the paper are converted to be dimensionless. The characteristic quantities used for nondimensionalization are fluid mass density, vein diameter, and the pulsatile period. Note that a unique feature 
of our studies is that, instead of using fixed data for a specific patient, we use dimensionless "ideal" data which are variables (including physiological values). Our purpose is to investigate the effects of these variables, because they may differ from patient to patient, and from time to time for a given patient.

Table 1: Parameters for All Simulations

\begin{tabular}{|c|c|c|c|c|c|c|c|c|c|}
\hline Group & Ratio & Angle & $\mathrm{Re}$ & $\mathrm{Kb}(\mathrm{V})$ & $\mathrm{Kb}(\mathrm{G})$ & $\mathrm{Ks}(\mathrm{V})$ & $\mathrm{Ks}(\mathrm{G})$ & $\operatorname{Inlet}(\mathrm{V})$ & $\mathrm{Inlet}(\mathrm{G})$ \\
\hline$(1)$ & $\mathbf{2 : 3}$ & 45 & 100 & 0.005 & 0.006 & 20 & 30 & 0.244 & $0.5 \vec{s}_{a}$ \\
fixed & $\mathbf{4 : 5}$ & 45 & 100 & 0.005 & 0.006 & 20 & 30 & 0.244 & $0.5 \vec{s}_{a}$ \\
speed & $\mathbf{1 : 1}$ & 45 & 100 & 0.005 & 0.006 & 20 & 30 & 0.244 & $0.5 \vec{s}_{a}$ \\
\hline$(1)$ & $\mathbf{2 : 3}$ & 45 & 100 & 0.005 & 0.006 & 20 & 30 & 0.174 & $0.5 \vec{s}_{a}$ \\
fixed & $\mathbf{4 : 5}$ & 45 & 100 & 0.005 & 0.006 & 20 & 30 & 0.174 & $0.347 \vec{s}_{a}$ \\
influx & $\mathbf{1 : 1}$ & 45 & 100 & 0.005 & 0.006 & 20 & 30 & 0.174 & $0.222 \vec{s}_{a}$ \\
\hline$(2)$ & $1: 1$ & $\mathbf{3 0}$ & 100 & 0.005 & 0.006 & 20 & 30 & 0.244 & $0.5 \vec{s}_{a}$ \\
& $1: 1$ & $\mathbf{4 5}$ & 100 & 0.005 & 0.006 & 20 & 30 & 0.244 & $0.5 \vec{s}_{a}$ \\
& $1: 1$ & $\mathbf{6 0}$ & 100 & 0.005 & 0.006 & 20 & 30 & 0.244 & $0.5 \vec{s}_{a}$ \\
\hline \multirow{2}{*}{$(3)$} & $4: 5$ & 45 & $\mathbf{5 0}$ & 0.005 & 0.006 & 20 & 30 & 0.244 & $0.5 \vec{s}_{a}$ \\
& $4: 5$ & 45 & $\mathbf{1 0 0}$ & 0.005 & 0.006 & 20 & 30 & 0.244 & $0.5 \vec{s}_{a}$ \\
& $4: 5$ & 45 & $\mathbf{1 5 0}$ & 0.005 & 0.006 & 20 & 30 & 0.244 & $0.5 \vec{s}_{a}$ \\
\hline \multirow{2}{*}{$(4)$} & $1: 1$ & 60 & 100 & 0.005 & 0.006 & 20 & 30 & 0.100 & $s_{a}(t)$ \\
& $1: 1$ & 60 & 100 & 0.005 & 0.006 & 20 & 30 & 0.380 & $s_{a}(t)$ \\
& $1: 1$ & 60 & 100 & 0.005 & 0.006 & 20 & 30 & 0.488 & $s_{a}(t)$ \\
& $1: 1$ & 60 & 100 & 0.005 & 0.006 & 20 & 30 & 0.600 & $s_{a}(t)$ \\
\hline
\end{tabular}

\subsection{Diameter ratio of graft to vein $\left(R_{g v}\right)$}

In dialysis practice the AVG has different diameter ranging between $4 \mathrm{~mm}$ to $8 \mathrm{~mm}$ [68]. The vein selected for AVG implantation has different size for different patients. Therefore the graft-to-vein diameter ratio $\left(R_{g v}\right)$ varies from case to case. This section addresses the possible effect of this ratio on the flow and force fields. Three different values are used: $R_{g v}=2: 3(0.67), 4: 5(0.80)$, 
and $1: 1(1.00)$. All other parameters are fixed, e.g., Reynolds number and attaching angle are $R e=100$ and $\theta=\frac{\pi}{4}$. See Table 1 for specific parameters used in this series of simulations. Since the blood flow influx at the AVG inlet depends on both the dialysis machine and blood flow from the artery, the influx is a complex unknown variable which is hard to control. Therefore, two ideal cases are considered in our work: fixed inlet speed (varying influx) and fixed inlet influx.

\subsubsection{Fixed inlet speed}

Figure 6 plots the contours of velocity (left column) and vorticity (right column) magnitudes on the plane perpendicular to the y-axis for three values of diameter ratio $\left(R_{g v}=2 / 3\right.$ for the first row, $4 / 5$ for the second row, and $1 / 1$ for the third row). From these figures we see that, as the diameter ratio increases, the flows in the graft are somewhat different; but the flows in the vein are quite similar. The former is caused by the enlarged flow domain due to the greater graft diameter and the increased flow influx into the system from the graft inlet due to fixed inlet speed. However, the graft is deformable and it distends to adjust to the increased fluid volume. Therefore the flow disturbances caused by the graft diameter and influx at inlet seem to be "absorbed" by the deformation of the graft. Consequentially, the flow fields in the vein look quite similar in the three cases.

We notice that during dialysis period, the flow and force fields may be very different from what has been observed in our simulations because Re is much higher. Higher Re indicates the inertia effect may dominate the viscous effect and more intensive flow structure may emerge. For instance, more vorticity may be generated and more vortices may be seen. However, since the dialysis period is much shorter than non-dialysis period, the possibly substantial difference may be only transitional.

Figure 7 plots the distributions of WSS (left column) and WNSG (right column) on the graft and vein walls (distribution of WSSG is similar). The first, second, and third rows correspond to $R_{g v}=2 / 3,4 / 5$, and $1 / 1$, respectively. By 
the same token, differences can be observed in the distributions on the graft among the three cases, but no apparent differences in the vein can be identified.

We point out that th£WSS maps in Fig. 7 look noisy in the graft. This may be caused by the fact that the graft wall (vein wall as well) is fastened to virtual springs immersed in viscous fluid to model the surrounding tissue. We are not much concerned by this oscillatory effect because: 1) The virtual springs modeling the tissue have the same elastic property in all of the simulations presented in the paper and the oscillatory effect would be the same for all simulations in all cases. 2) If the oscillatory effect would dominate in the simulations, then one would expect similar oscillatory effect would occur in the vein as well. But this is not the case as shown in Fig. 7.

Table 2: Spatially averaged WSS, WSSG, and WNSG for different diameter ratios (equal speed).

\begin{tabular}{|c||c|c||c|c||c|c|}
\hline$R_{g v}$ & WSS(V) & WSS(G) & WSSG(V) & WSSG(G) & WNSG(V) & WNSG(G) \\
\hline $2: 3$ & 2.44 & 29.85 & 22.73 & 513.57 & 2.12 & 51.55 \\
$4: 5$ & 2.53 & 25.39 & 23.27 & 435.19 & 2.46 & 51.88 \\
$1: 1$ & 2.62 & 23.13 & 25.97 & 366.09 & 2.46 & 41.55 \\
\hline
\end{tabular}

The spatially averaged WSS, WSSG, and WNSG on the graft (denoted by G) and vein (denoted by V) are computed aㅔ listed on Table 2. The gradients are computed along the longitudinal and the circumferential directions. The WSSG and WNSG are magnitudes of the gradients of WSS and WNS. The WNS includes both the pressure and the viscous contributions. These apply to all of the tables listing the WSS, WSSG, and WNSG in the paper. The table shows that these variables increase in the vein but decreases in the graft as the ratio increases. The former is caused by the increased flow flux from the graft and the latter may be caused by the increased dilation of the graft as the ratio increases (induced by enlarged inlet influx and graft diameter). 
We notice that the averaged WSS, WSSG, and WNSG in the graft are significantly greater than in the vein.

We point out that another important quantity is the oscillatory shear index (OSI), as defined in $[38,37]$, which is used to gauge the bi-directionality of the WSS caused by reverse flows. As was mentioned in [44] the influence of flow pulsatility is substantially reduced in the compliant model compared to the rigid model. And no significant reverse flows (primary or secondary) are observed in our simulations. Therefore, the OSI should be zero in our cases. This is consistent with the results from McNally et al. [37].

\subsubsection{Fixed influx}

As the graft-to-vein diameter ratio changes, the fluid influx at the graft inlet changes too since the inlet velocity profile is fixed. To make the influx equal for all the three different ratios, the inlet velocity profile is adjusted in such a way that the influx at the graft inlet is the same in all the cases of different ratios. A series of simulations are performed.

Two typical results are shown below. The left bolumn in Figure 8 shows contours of velocity magnitude on the plane normal to the y-axis; the right column shows the distribution of WSS on the graft-vein wall. One can see obvious differences in the flow fields in the graft for the three different ratios. However, only slight differences in the vein are discernable: the flows look quite similar in the vein. For the distribution of WSS (similar for WSSG/WNSG), only noticeable differences are seen in the graft; the distribution looks quite the same in the vein for all the three ratios.

Table 3 shows the averaged WSS, WSSG, and WNSG on the graft and vein walls for different ratios. We see that almost all of these averaged quantities decrease in the graft and vein as the ratio increases. The decrease is probably caused by the slowed-down flow in the graft-vein system because of the fixed influx at the graft inlet and the increased graft diameter. We also notice that the WSS, WSSG, and WNSG in the graft are all greater than those in the vein.

We notice that the WSS, WSSG, and WNSG in the fixed inlet speed case 
are almost all greater than in the fixed inlet flux case. The enhanced flow influx in the former case contributes to the increase in the three quantities.

To summarize, our computational results of different diameter ratios show that: as the graft-vein diameter ratios increases, 1) the flow fields in the graft become different but they remain almost the same in the vein; 2) the WSS, WSSG, WNSG, and their averaged values in the vein all increase in the fixed inlet speed case, but decreases in the fixed influx case. In both cases, they decrease in graft. 3) The averaged WSS, WSSG, and WNSG in the graft are significantly greater than in the vein.

These are partially illustrated giphically by Fig. 3 .

\subsection{Attaching Angle}

In this section, AVG configurations with different attaching angles $\theta\left(30^{\circ}\right.$, $45^{\circ}$, and $60^{\circ}$ ) are compared. All simulations reported below are conducted with Reynolds number of 100 and diameter ratio of 1:1. Other parameters are listed in Table 1. Notice that other ratio (e.g., 2:3) and vein inlet-speed are used as well. Computational results are similar.

Figure 9 visualizes the contours of velocity (left column) and vorticity (right column) in magnitude on the plane normal to the y-axis. From this figure, one can see that the major difference in the flow fields lies in the graft and no apparent difference is observable in the vein for the three attaching angles. This is attributed to the fact that the flow disturbances induced by different attaching

Table 3: Spatially averaged WSS, WSSG, and WNSG for different diameter ratios (equal influx).

\begin{tabular}{|c||c|c||c|c||c|c|}
\hline$R_{g v}$ & WSS(V) & WSS(G) & WSSG(V) & WSSG(G) & WNSG(V) & WNSG(G) \\
\hline $2: 3$ & 1.65 & 30.22 & 15.42 & 522.31 & 1.47 & 50.54 \\
$4: 5$ & 1.48 & 22.13 & 13.78 & 380.43 & 1.35 & 31.52 \\
$1: 1$ & 1.38 & 12.39 & 13.83 & 196.42 & 1.19 & 19.09 \\
\hline
\end{tabular}


angles are taken in by the graft and "annihilated" by the elastic deformation of the graft.

Figure 10 plots the distributions of wall shear stress (left column) and wall normal stress gradient (right column) for the three different angles (the first, second and third rows correspond to $\theta=30^{\circ}, 45^{\circ}, 60^{\circ}$, respectively). This figure shows that while differences on the graft are discernable, differences on the vein are not obvious. This is due to the graft deformability which can lessen flow disturbances induced by varying attaching angle.

Table 4: Spatially averaged WSS, WSSG, and WNSG for different attaching angles.

\begin{tabular}{|c||c|c||c|c||c|c|}
\hline$\theta$ & WSS(V) & WSS(G) & WSSG(V) & WSSG(G) & WNSG(V) & WNSG(G) \\
\hline 30 & 3.01 & 26.51 & 29.20 & 415.63 & 3.08 & 31.96 \\
45 & 3.05 & 22.38 & 29.12 & 305.79 & 3.09 & 22.67 \\
60 & 3.04 & 20.28 & 29.33 & 268.68 & 3.01 & 19.00 \\
\hline
\end{tabular}

Table 4 lists the averaged WSS, WSSG, and WNSG on the graft and vein for three different angles. It is seen that these quantities are greater in the graft than in the vein. They all decrease in the graft, but remain roughly the same in the vein for different attaching angles. The former is caused by the increasing attaching angle. The greater the angle, the less aligned the flow in the graft is to the flow at inlet, and the more flow disturbance is induced in the graft, thus resulting in less forces such as WSS. The latter is caused by the graft deformability which may diminish the flow disturbances.

The effect of attaching angle is summarized as follows. As the attaching angle increases, 1)the flow field becomes different in the graft, but remains roughly the same in the vein; 2)the WSS, WSSG, WNSG, and their averaged values decrease in the graft but remain nearly the same in the vein. 3) The averaged WSS, WSSG, and WNSG in the graft are significantly greater than those in the vein. 
These are partially illustrated gaphically by Fig. 4 (left panel).

\subsection{Reynolds Number}

This section investigates Reynolds number effects on flow and force fields in the graft-ein system. Figure 11 plots the contours of velocity (left column) and vorticity (right column) magnitudes on the plane perpendicular to the y-axis for three different Reynolds numbers (the first, second, and third rows correspond to $R e=50,100,150$, respectively). From this figure and more visualizations not shown here we find that as Re increases the velocity/vorticity magnitudes become larger and more flow patterns and vortices are seen. This is because bigger Re corresponds to smaller viscous force and larger inertia force. Therefore the flow gets sped up more easily and forms more flow structures.

Figure 12 plots the WSS (left column) and WNSG (right column). The distributions of these variables are slightly different on the graft and are quite similar on the vein for the three Reynolds numbers. Table 5 lists the averaged WSS, WSSG, and WNGS in the graft and vein. We see that as Re increases, these quantities change slightly, particularly in the vein. Presumably the decreased viscous force as Re increases may generate smaller WSS. But according to our simulations, it is not the case. This may be explained by the graft/vein flexibility that decreases the Re-induced flow disturbances. Again WSS, WSSG, and WNSG are all greater in the graft than in the vein.

To summarize the Re effect, our simulations indicate that as Re increases, 1) velocity and vorticity magnitudes become greater and more complex flow patterns are formed in the graft-vein system; 2) the WSS, WSSG, WNSG and their spatially averaged values change slightly; 3) the averaged WSS, WSSG, and WNSG in the graft are significantly greater than in the vein.

These are partially illustrated gaphically by Fig. 4 (right panel).

\subsection{Vein inlet speed}

In all of the above simulations, the speed of blood flow at the vein inlet is fixed. But in dialysis the blood speed in the vein may be different for different 
Table 5: Spatially averaged WSS, WSSG, and WNSG for different Reynolds numbers.

\begin{tabular}{|c||c|c||c|c||c|c|}
\hline Re & WSS(V) & WSS(G) & WSSG(V) & WSSG(G) & WNSG(V) & WNSG(G) \\
\hline 50 & 2.51 & 28.67 & 21.95 & 491.97 & 2.42 & 51.62 \\
100 & 2.53 & 25.39 & 23.27 & 435.19 & 2.46 & 51.88 \\
150 & 2.50 & 25.82 & 23.56 & 437.27 & 2.35 & 52.53 \\
\hline
\end{tabular}

patient and different at different times (e.g., when walking or resting). To investigate possible effect of this speed, four different values of vein inlet speed (dimensionless) are used $V_{v}=0.1,0.38,0.488,0.6$. The cases with the lowest and the highest speeds are reported below.

Figure 13 plots the contours of velocity (first row) and vorticity (second row) magnitudes on a few planes normal to the x-axis for the lowest (left column) and highest (right column) vein inlet speed. From this figure, one can see more vortical structures in the vein at the high speed case which indicates the higher vein inlet-speed generates more vorticity and induces more vortices in the vein. The flow field in the graft is almost the same since the flow in the vein does not affect the flow in the graft except at the conjunction. It is also noticed that the vein looks slightly enlarged (its diameter becomes a little bigger), particularly at the section near the inlet.

Figure 14 plots the WSS (first row) and WNSG (second row) for the lowest (left column) and highest (right column) vein inlet speeds. From these figures one can see that the WSS and WNSG on the vein wall are higher in the high speed case. This is because the faster fluid flow in the vein induces greater shear stress on the vein wall. The distributions of these variables on the graft are quite similar.

Table 6 shows the averaged WSS, WSSG, and WNSG on the graft and vein for different vein inlet speed. We see that as the speed increases, all of these quantities increases in the vein. But WSS and its gradient decrease and WNSG 
increases in the graft. Again, these quantities in the graft are significantly greater than in the vein.

Table 6: Spatially averaged WSS, WSSG, and WNSG for different vein inlet speed.

\begin{tabular}{|c||c|c||c|c||c|c|}
\hline$U_{v}$ & WSS(V) & WSS(G) & WSSG(V) & WSSG(G) & WNSG(V) & WNSG(G) \\
\hline 0.100 & 3.03 & 47.81 & 29.66 & 803.30 & 3.62 & 102.08 \\
0.380 & 6.84 & 48.04 & 66.52 & 797.92 & 6.44 & 115.33 \\
0.488 & 8.27 & 47.80 & 76.53 & 790.25 & 8.17 & 119.37 \\
0.600 & 9.82 & 47.46 & 86.22 & 782.16 & 9.13 & 123.40 \\
\hline
\end{tabular}

The effect of vein inlet speed is summarized as follows. As the vein inletspeed increases, 1) more complex flow patterns (vortices) and more radial vein expansion are induced in the vein, but flow in the graft is not affected; 2) the WSS, WSSG, WNSG, and their averaged values increase in the vein but the averaged WSS/WSSG decrease and WNSG increases in the graft; 3)the averaged WSS, WSSG, and WNSG in the graft are significantly greater than in the vein.

These are partially illustrated gfaphically by Fig. 5 .

\section{Summary and Conclusion}

To summarize, numerous simulations are designed and performed on blood flow through a distal compliant AVG anastomosis model with various combinations of dimensionless values of the AVG diameter ratio $R_{g v}$, attaching angle $\theta$, flow Reynolds number $R e$, and vein inlet speed $V_{v}$. The flow and force fields are visualized and analyzed. The major conclusions are as follows. 1) The influence of the three dimensionless parameters $\left(R_{g v}, \theta\right.$, and $\left.R e\right)$ on the flow and force fields lie largely on the graft. Their influences on the vein are less distinct: as Re increases, more complex flow patterns are formed; as the AVG diameter ratio increases, the WSS, WSSG, and WNSG and their averaged values increase 
at the fixed speed case and decrease at the fixed influx case. The significantly reduced effect is caused by the flexibility of the graft and vein. 2) The vein inlet speed has substantial influence on the flow and force fields in the vein, but not in the draft. The flow becomes more complex and WSS, WSSG, and WNSG all increase in the vein as vein inlet speed increases. 3) In all cases, the WSS, WSSG, and WNSG and their averaged values on the graft are significantly greater than in the vein.

\section{Acknowledgments}

We would like to thank the unknown Referees for their comments and suggestions which have helped us in preparing a better presentation of our work. This work was supported by the National Science Foundation (DMS-1522554). All authors declare no conflict of interest related to this work.

[1] S. J. Schwab, Vascular access for hemodialysis, Kidney international 55 (5) (1999) 2078-2090.

[2] E. C. Kovalik, S. J. Schwab, Can venous stenosis be prevented?, in: Seminars in Dialysis, Vol. 12, Wiley Online Library, 1999, pp. 144-145.

[3] A. Brahmbhatt, A. Remuzzi, M. Franzoni, S. Misra, The molecular mechanisms of hemodialysis vascular access failure, Kidney international 89 (2) (2016) 303-316.

[4] P. Budu-Grajdeanu, R. C. Schugart, A. Friedman, C. Valentine, A. K. Agarwal, B. H. Rovin, A mathematical model of venous neointimal hyperplasia formation, Theoretical Biology and Medical Modelling 5 (1) (2008) 2 .

[5] P. E. Miller, D. Carlton, M. H. Deierhoi, D. T. Redden, M. Allon, Natural history of arteriovenous grafts in hemodialysis patients, American journal of kidney diseases 36 (1) (2000) 68-74.

[6] M. Lemson, J. Tordoir, M. Daemen, P. Kitslaar, Intimal hyperplasia in vascular grafts, European Journal of Vascular and Endovascular Surgery 19 (4) (2000) 336-350. 
[7] H. Haruguchi, S. Teraoka, Intimal hyperplasia and hemodynamic factors in arterial bypass and arteriovenous grafts: a review, Journal of Artificial Organs 6 (4) (2003) 227-235.

[8] C. V. Cunnane, E. M. Cunnane, M. T. Walsh, A review of the hemodynamic factors believed to contribute to vascular access dysfunction, Cardiovascular engineering and technology 8 (3) (2017) 280-294.

[9] R. S. Reneman, T. Arts, A. P. Hoeks, Wall shear stress-an important determinant of endothelial cell function and structure-in the arterial system in vivo, Journal of vascular research 43 (3) (2006) 251-269.

[10] O. Traub, B. C. Berk, Laminar shear stress: mechanisms by which endothelial cells transduce an atheroprotective force, Arteriosclerosis, thrombosis, and vascular biology 18 (5) (1998) 677-685.

[11] P. F. Davies, A. Remuzzi, E. J. Gordon, C. F. Dewey, M. A. Gimbrone, Turbulent fluid shear stress induces vascular endothelial cell turnover in vitro, Proceedings of the National Academy of Sciences 83 (7) (1986) 21142117.

[12] P. Dobrin, F. Littooy, E. Endean, Mechanical factors predisposing to intimal hyperplasia and medial thickening in autogenous vein grafts., Surgery 105 (3) (1989) 393-400.

[13] M. Ojha, C. R. Ethier, K. W. Johnston, R. S. Cobbold, Steady and pulsatile flow fields in an end-to-side arterial anastomosis model, Journal of vascular surgery 12 (6) (1990) 747-753.

[14] L. W. Kraiss, T. R. Kirkman, T. R. Kohler, B. Zierler, A. W. Clowes, Shear stress regulates smooth muscle proliferation and neointimal thickening in porous polytetrafluoroethylene grafts., Arteriosclerosis, Thrombosis, and Vascular Biology 11 (6) (1991) 1844-1852. 
[15] M. E. Cinat, J. Hopkins, S. E. Wilson, A prospective evaluation of ptfe graft patency and surveillance techniques in hemodialysis access, Annals of vascular surgery 13 (2) (1999) 191-198.

[16] A. Mima, Hemodialysis vascular access dysfunction: molecular mechanisms and treatment, Therapeutic Apheresis and Dialysis 16 (4) (2012) 321-327.

[17] A. Clowes, M. Reidy, M. Clowes, Mechanisms of stenosis after arterial injury., Laboratory investigation; a journal of technical methods and pathology 49 (2) (1983) 208-215.

[18] M. F. Fillinger, E. R. Reinitz, R. A. Schwartz, D. E. Resetarits, A. M. Paskanik, D. Bruch, C. E. Bredenberg, Graft geometry and venous intimalmedial hyperplasia in arteriovenous loop grafts, Journal of Vascular Surgery 11 (4) (1990) 556-566.

[19] P. J. Brands, A. P. Hoeks, L. Hofstra, R. S. Reneman, A noninvasive method to estimate wall shear rate using ultrasound, Ultrasound in medicine and biology 21 (2) (1995) 171-185.

[20] M. J. Thubrikar, Vascular mechanics and pathology, Springer Science \& Business Media, 2007.

[21] S. Unnikrishnan, T. N. Huynh, B. Brott, Y. Ito, C. Cheng, A. Shih, M. Allon, A. S. Anayiotos, Turbulent flow evaluation of the venous needle during hemodialysis, Journal of biomechanical engineering 127 (7) (2005) 11411146.

[22] T. N. Huynh, B. K. Chacko, X. Teng, B. C. Brott, M. Allon, S. S. Kelpke, J. A. Thompson, R. P. Patel, A. S. Anayiotos, Effects of venous needle turbulence during ex vivo hemodialysis on endothelial morphology and nitric oxide formation, Journal of biomechanics 40 (10) (2007) 2158-2166.

[23] O. Kh. Jahrome, I. Hoefer, G. J. Houston, P. A. Stonebridge, P. J. Blankestijn, F. L. Moll, G. J. De Borst, Hemodynamic effects of spiral 
eptfe prosthesis compared with standard arteriovenous graft in a carotid to jugular vein porcine model, The journal of vascular access 12 (3) (2011) $224-230$.

[24] M. R. De Vries, P. H. Quax, inflammation in vein graft disease, Frontiers in Cardiovascular Medicine 5 (2018) 3.

[25] D. Fulker, B. Ene-Iordache, T. Barber, High-resolution computational fluid dynamic simulation of haemodialysis cannulation in a patient-specific arteriovenous fistula, Journal of biomechanical engineering 140 (3) (2018) 031011.

[26] L. Grechy, F. Iori, R. Corbett, S. Shurey, W. Gedroyc, N. Duncan, C. Caro, P. Vincent, Suppressing unsteady flow in arterio-venous fistulae, Physics of Fluids 29 (10) (2017) 101901.

[27] P. Longest, C. Kleinstreuer, Computational haemodynamics analysis and comparison study of arterio-venous grafts, Journal of medical engineering \& technology 24 (3) (2000) 102-110.

[28] F. Loth, P. F. Fischer, N. Arslan, C. D. Bertram, S. E. Lee, T. J. Royston, W. E. Shaalan, H. S. Bassiouny, Transitional flow at the venous anastomosis of an arteriovenous graft: potential activation of the erk1/2 mechanotransduction pathway, Journal of Biomechanical Engineering 125 (1) (2003) 49 61.

[29] J. Chen, X.-Y. Lu, W. Wang, Non-newtonian effects of blood flow on hemodynamics in distal vascular graft anastomoses, Journal of Biomechanics 39 (11) (2006) 1983-1995.

[30] S.-W. Lee, D. S. Smith, F. Loth, P. F. Fischer, H. S. Bassiouny, Importance of flow division on transition to turbulence within an arteriovenous graft, Journal of biomechanics 40 (5) (2007) 981-992.

[31] J. M. Ortega, W. Small, T. S. Wilson, W. J. Benett, J. M. Loge, D. J. Maitland, A shape memory polymer dialysis needle adapter for the reduc- 
tion of hemodynamic stress within arteriovenous grafts, IEEE Transactions on Biomedical Engineering 54 (9) (2007) 1722-1724.

[32] T. A. Manos, D. P. Sokolis, A. T. Giagini, C. H. Davos, J. D. Kakisis, E. P. Kritharis, N. Stergiopulos, P. E. Karayannacos, S. Tsangaris, Local hemodynamics and intimal hyperplasia at the venous side of a porcine arteriovenous shunt, IEEE Transactions on Information Technology in Biomedicine 14 (3) (2010) 681-690.

[33] H.-H. Kim, Y. H. Choi, S.-H. Suh, J. S. Lee, Y. H. Jung, Y. H. So, Arteriovenous graft modeling and hemodynamic interpretation, Open Journal of Fluid Dynamics 2 (04) (2012) 324.

[34] S. Khruasingkeaw, Y. Khunatorn, K. Rerkasem, T. Srisuwan, Wall shear stress distribution in arteriovenous graft anastomosis using computational fluid dynamics, International Journal of Pharma Medicine and Biological Sciences 5 (1) (2016) 71.

[35] D. Williams, Computational fluid dynamics analysis of arteriovenous graft configurations, Engineering and Applied Science Theses \& Dissertations. 340. Washington University in St. Louis.

[36] A. McNally, A. G. Akingba, E. A. Robinson, P. Sucosky, Novel modular anastomotic valve device for hemodialysis vascular access: preliminary computational hemodynamic assessment, The journal of vascular access 15 (6) (2014) 448-460.

[37] A. McNally, A. G. Akingba, P. Sucosky, Effect of arteriovenous graft flow rate on vascular access hemodynamics in a novel modular anastomotic valve device, The Journal of Vascular Access 19 (5) (2018) 446-454.

[38] B. Ene-Iordache, A. Remuzzi, Blood flow in idealized vascular access for hemodialysis: a review of computational studies, Cardiovascular engineering and technology 8 (3) (2017) 295-312. 
[39] S. Vogel, Life in moving fluids: the physical biology of flow, Princeton University Press, 1996.

[40] A. Voorhees, G. B. Nackman, T. Wei, Experiments show importance of flow-induced pressure on endothelial cell shape and alignment, in: Proceedings of the Royal Society of London A: Mathematical, Physical and Engineering Sciences, Vol. 463, The Royal Society, 2007, pp. 1409-1419.

[41] S. Pope, Turbulent Flows, 771 pp, Cambridge Univ. Press, Cambridge, UK, 2000.

[42] I. Decorato, Z. Kharboutly, C. Legallais, A.-V. Salsac, Numerical study of the influence of wall compliance on the haemodynamics in a patient-specific arteriovenous fistula, Computer Methods in Biomechanics and Biomedical Engineering 14 (sup1) (2011) 121-123.

[43] P. M. McGah, D. F. Leotta, K. W. Beach, A. Aliseda, Effects of wall distensibility in hemodynamic simulations of an arteriovenous fistula, Biomechanics and modeling in mechanobiology 13 (3) (2014) 679-695.

[44] Z. Bai, L. Zhu, Three-dimensional simulation of a viscous flow past a compliant model of arteriovenous-graft anastomosis, Computers \& Fluids 181 (2019) 403-415.

[45] C. S. Peskin, The immersed boundary method, Acta numerica 11 (2002) $479-517$.

[46] W. Quanyu, L. Xiaojie, P. Lingjiao, T. Weige, Q. Chunqi, Simulation analysis of blood flow in arteries of the human arm, Biomedical Engineering: Applications, Basis and Communications 29 (04) (2017) 1750031.

[47] M. W. Roos, E. Wadbro, M. Berggren, Computational estimation of fluid mechanical benefits from a fluid deflector at the distal end of artificial vascular grafts, Computers in biology and medicine 43 (2) (2013) 164-168. 
[48] M. Bukač, S. Čanić, J. Tambača, Y. Wang, Fluid-structure interaction between pulsatile blood flow and a curved stented coronary artery on a beating heart: A four stent computational study, Computer Methods in Applied Mechanics and Engineering.

[49] Y. Wu, X.-C. Cai, A parallel two-level method for simulating blood flows in branching arteries with the resistive boundary condition, Computers \& Fluids 45 (1) (2011) 92-102.

[50] P. F. Fischer, F. Loth, S. E. Lee, S.-W. Lee, D. S. Smith, H. S. Bassiouny, Simulation of high-reynolds number vascular flows, Computer methods in applied mechanics and engineering 196 (31-32) (2007) 3049-3060.

[51] Z. Guo, C. Shu, Lattice Boltzmann method and its applications in engineering, Vol. 3, World Scientific, 2013.

[52] S. Succi, The Lattice Boltzmann Equation: For Complex States of Flowing Matter, Oxford University Press, 2018.

[53] W. Strychalski, C. A. Copos, O. L. Lewis, R. D. Guy, A poroelastic immersed boundary method with applications to cell biology, Journal of Computational Physics 282 (2015) 77-97.

[54] Y. H. Qian, Lattice gas and lattice kinetic theory applied to the navierstokes equations, Doktorarbeit, Universite Pierre et Marie Curie, Paris.

[55] C. SY, D. GD, Lattice boltzmann method for fluid flows, Annual Review of Fluid Mechanics 30 (1998) 329.

[56] L.-S. Luo, Unified theory of lattice Boltzmann models for nonideal gases, Physical review letters 81 (8) (1998) 1618.

[57] D. A. Wolf-Gladrow, Lattice-gas cellular automata and lattice Boltzmann models: An Introduction, no. 1725, Springer, 2000.

[58] H. Huang, M. Sukop, X. Lu, Multiphase lattice Boltzmann methods: Theory and application, John Wiley \& Sons, 2015. 
[59] T. Krüger, H. Kusumaatmaja, A. Kuzmin, O. Shardt, G. Silva, E. M. Viggen, The lattice boltzmann method, Springer International Publishing 10 (2017) 978-3.

[60] Z. Guo, C. Zheng, B. Shi, Discrete lattice effects on the forcing term in the lattice boltzmann method, Physical Review E 65 (4) (2002) 046308.

[61] L. Zhu, G. He, S. Wang, L. Miller, X. Zhang, Q. You, S. Fang, An immersed boundary method based on the lattice boltzmann approach in three dimensions, with application, Computers \& Mathematics with Applications 61 (12) (2011) 3506-3518.

[62] L. Zhu, X. Yu, N. Liu, Y. Cheng, X. Lu, A deformable plate interacting with a non-newtonian fluid in three dimensions, Physics of Fluids 29 (8) (2017) 083101.

[63] L. Zhu, A new 3d immersed boundary method for non-newtonian fluidstructure-interaction with application, Theoretical and Applied Mechanics Letters 8 (3) (2018) 193-196.

[64] B. Zigon, L. Zhu, F. Song, Interactive 3d simulation for fluid-structure interactions using dual coupled gpus, The Journal of Supercomputing 74 (1) (2018) 37-64.

[65] Z. Bai, Modeling and simulation of blood flow past the distal anastomosis of arteriovenous graft for hemodialysis, PhD Dissertation, Department of Mathematical Sciences, Indiana University - Purdue University Indianapolis.

[66] S. Misra, A. A. Fu, A. Puggioni, K. M. Karimi, J. N. Mandrekar, J. F. Glockner, L. A. Juncos, B. Anwer, A. M. McGuire, D. Mukhopadhyay, Increased shear stress with upregulation of vegf-a and its receptors and mmp2, mmp-9, and timp-1 in venous stenosis of hemodialysis grafts, American Journal of Physiology-Heart and Circulatory Physiology 294 (5) (2008) H2219-H2230. 
[67] T. G. Papaioannou, C. Stefanadis, Vascular wall shear stress: basic principles and methods, Hellenic J Cardiol 46 (1) (2005) 9-15.

[68] M. J. Oliver, S. Schwab, A. Sheridan, Chronic hemodialysis vascular access: Types and placement, UpToDate. Version 13.

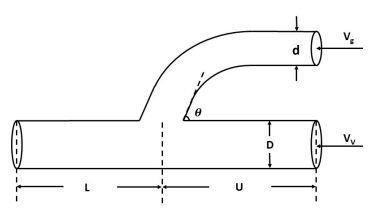

(a) Anastomosis Model

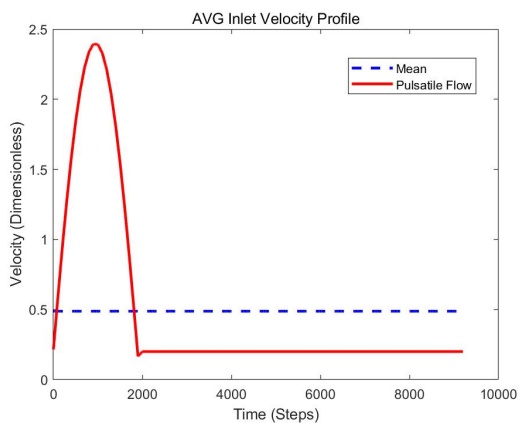

(b) Velocity Profile at AVG Inlet

Figure 1: Diagram of the anastomosis model (subfigure a) and velocity profile at the graft inlet (subfigure b). The $\mathrm{x}$-axis points from left to right. The $\mathrm{y}$-axis points from front to rear, perpendicular to the paper. The z-axis points from bottom to top. Pulsatile velocity $V_{g}$ is imposed on the graft inlet. Constant velocity $V_{v}$ is imposed on the vein inlet. No friction boundary condition is used on the vein outlet. The symbol $\theta$ is the graft attaching angle defined as the angle between the $x$-axis and the tangent to the graft at the vein-graft intersection point on the middle $x-z$ plane along $y$ direction. The letters $d$ and $D$ are the diameters of the graft and the vein, respectively. The distances $\mathrm{L}$ and $\mathrm{U}$ are set as $8 \mathrm{D}$. The entire anastomosis model is placed in the center (along all directions) of a rectangular computational domain of stationary viscous fluid. The right panel plots the AVG inlet velocity. The $x$-axis is the dimensionless time (i.e. steps in LB simulation). The $y$-axis is the dimensionless velocity magnitude. The dashed line is the mean speed over a cycle. 


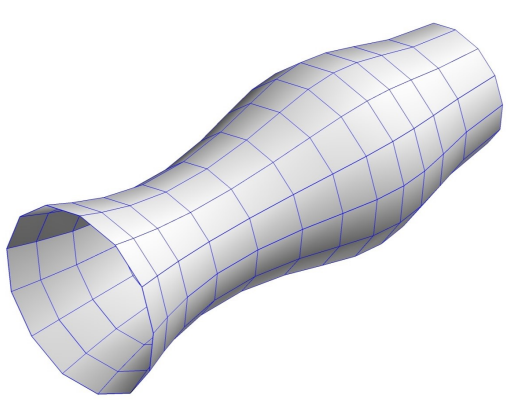

(a) grid size $10^{3}$

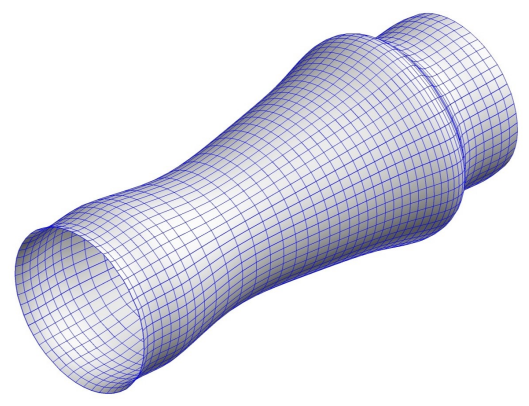

(c) grid size $40^{3}$

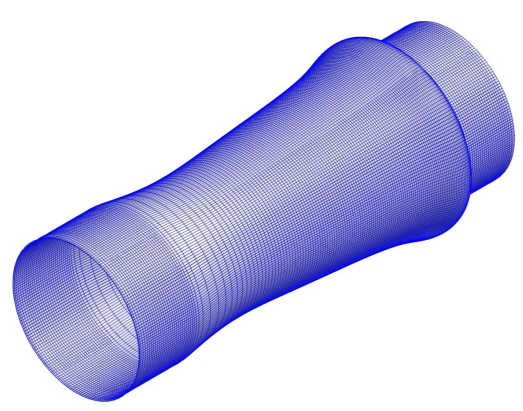

(e) grid size $160^{3}$

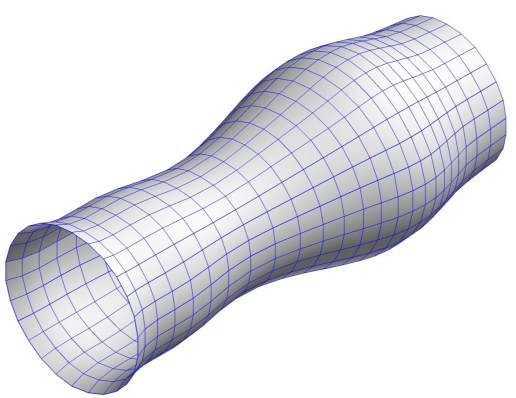

(b) grid size $20^{3}$

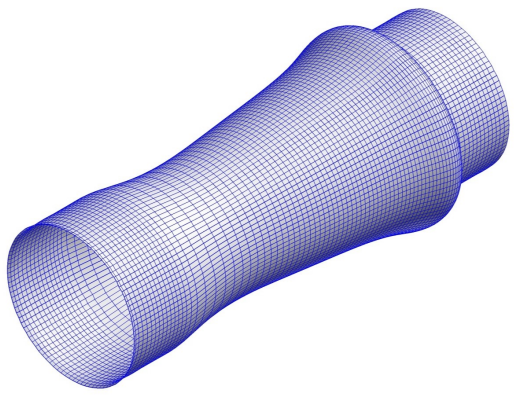

(d) grid size $80^{3}$

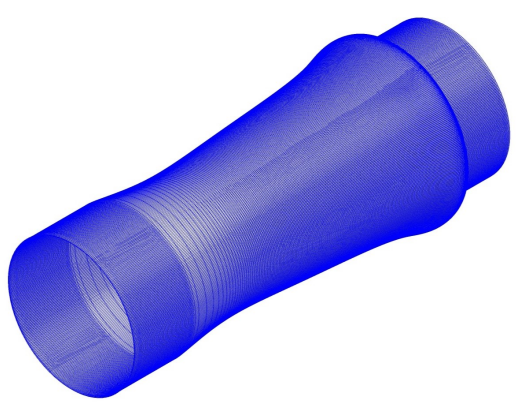

(f) grid size $320^{3}$

Figure 2: Mesh refinement study for a viscous fluid flowing in a flexible tube immersed in a stationary fluid. The figure plots the shape/position of the tube surface obtained on six fluid grids: $10^{3}, 20^{3}, 40^{3}, 80^{3}, 160^{3}, 320^{3}$ (subfigures a, b, c, d, e, f, respectively). One can see that the shape/position are already converged on the grid of $80^{3}$ nodes. 


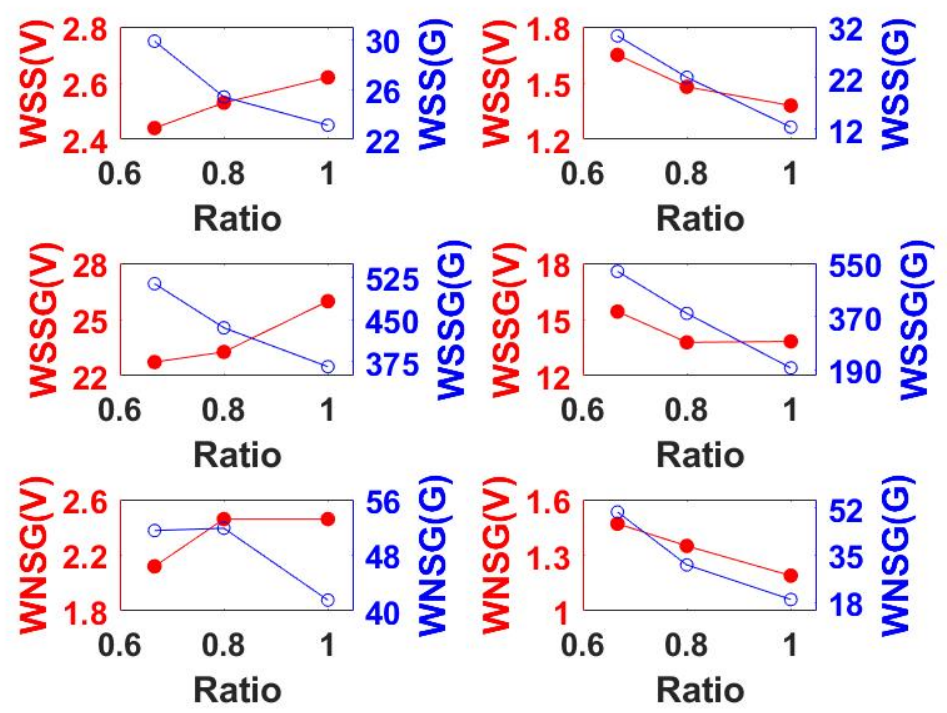

Figure 3: Effect of diameter ratio $R_{g v}$. Left panel is the fixed speed case and the right panel is the fixed influx case. The first row plots WSS, the second row plots WSSG, and the third row plots WNSG. In each subfigure, the left y-axis corresponds to data on the vein (in red) and the right $\mathrm{y}$-axis corresponds to data in the graft (in blue). The $\mathrm{x}$-axis shows the ratio. 

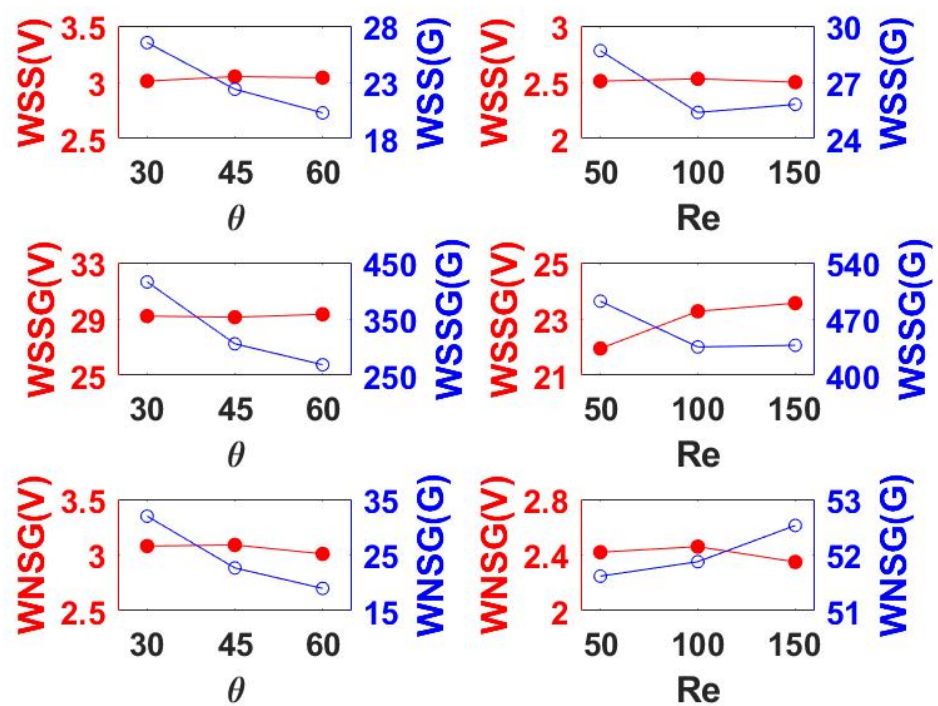

Figure 4: Effects of attaching angle $\theta$ (left panel) and Reynolds number Re (right panel). The first row plots WSS, the second row plots WSSG, and the third row plots WNSG. In each subfigure, the left y-axis corresponds to data on the vein (in red) and the right y-axis corresponds to data in the graft (in blue). The $\mathrm{x}$-axis plots the attaching angle in the left panel and plots the Reynolds number in the right panel.

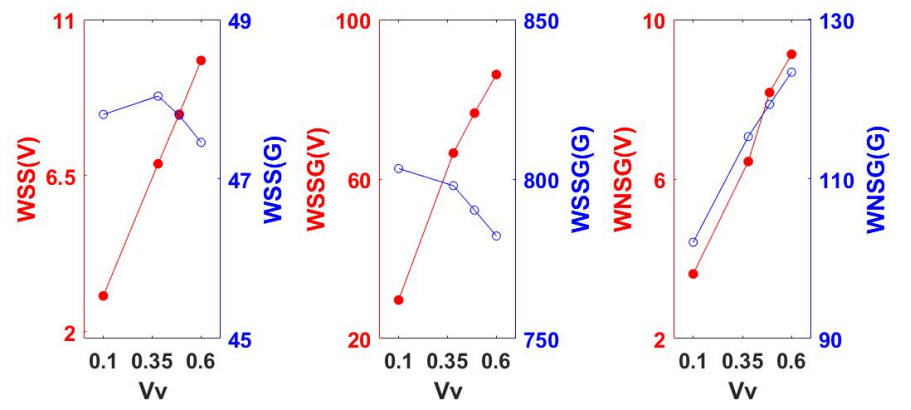

Figure 5: Effect of vein inlet speed $\left(V_{v}\right)$. The first panel plots WSS, the second panel plots WSSG, and the third panel plots WNSG. In each subfigure, the left y-axis corresponds to data on the vein (in red) and the right y-axis corresponds to data in the graft (in blue). The $\mathrm{x}$-axis shows the vein inlet speed. 


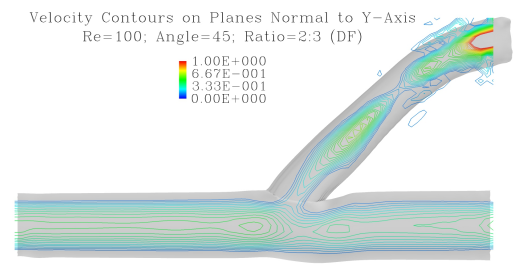

(a) Ratio $=2: 3$

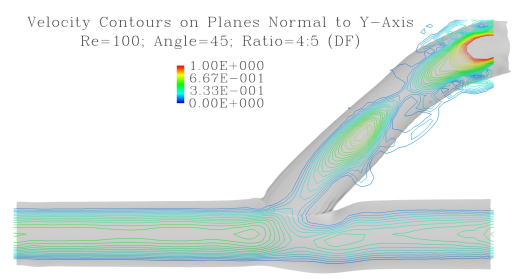

(c) Ratio $=4: 5$

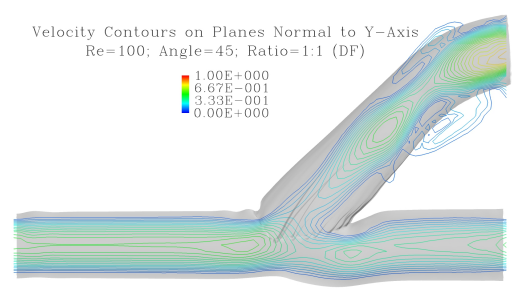

(e) Ratio=1:1

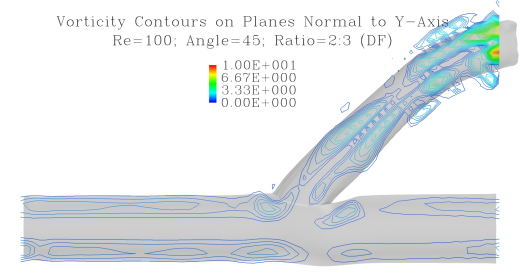

(b) Ratio $=2: 3$

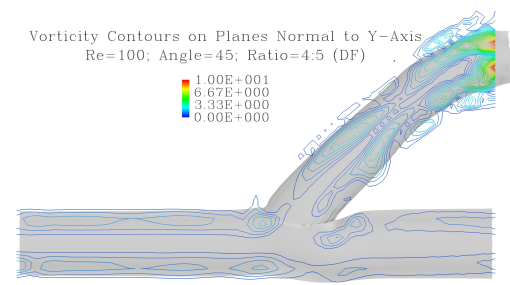

(d) Ratio=4:5

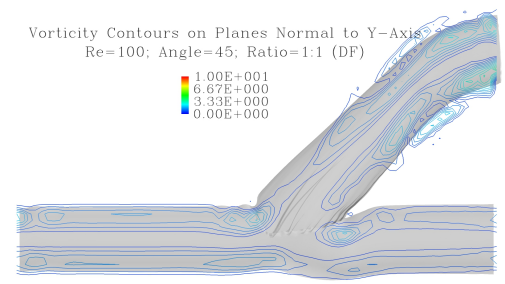

(f) Ratio=1:1

Figure 6: Effect of graft-vein diameter ratio (fixed graft-inlet flow-speed). Contours of velocity (subfigures a, c, e) and vorticity (subfigures b, d, f) $\perp$ Y-Axis with different ratios: $R_{g v}=2: 3$ (subfigures a, b), $4: 5$ (subfigures c, d) , $1: 1$ (subfigures e, f). Other parameters are fixed: $R e=100, \theta=\pi / 4, V_{v}=0.244$. 


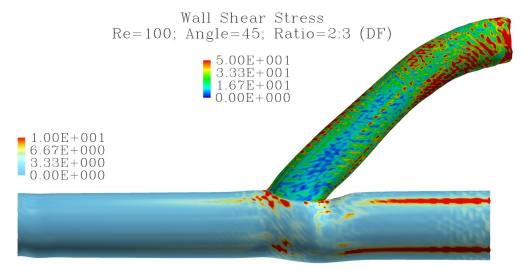

(a) Ratio $=2: 3$

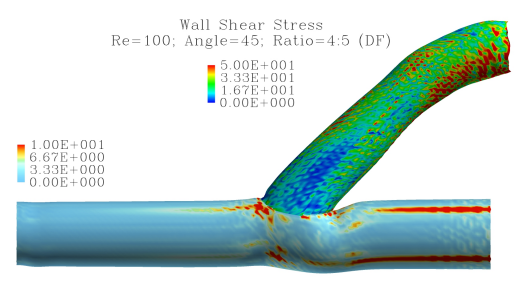

(c) Ratio $=4: 5$

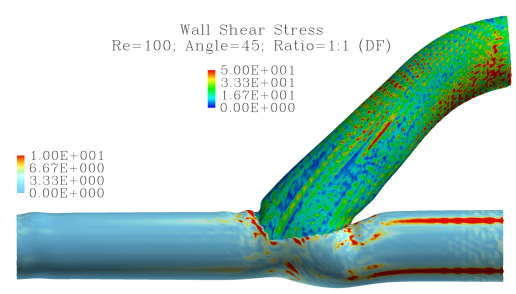

(e) Ratio=1:1

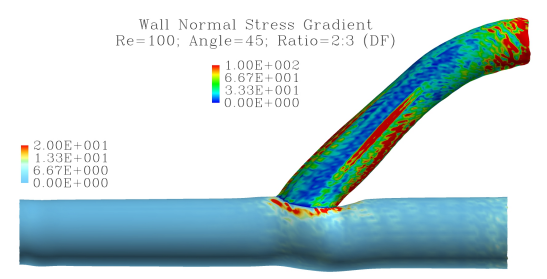

(b) Ratio $=2: 3$

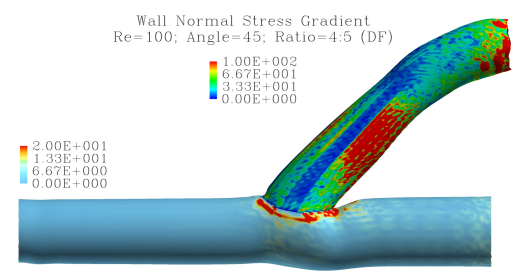

(d) Ratio $=4: 5$

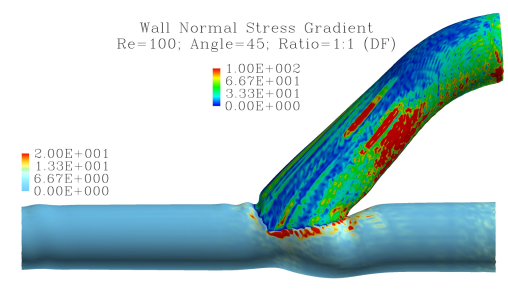

(f) Ratio $=1: 1$

Figure 7: Effect of graft-vein diameter ratio (fixed graft-inlet flow-speed). Distributions of WSS (subfigures a, c, e) and WNSG (subfigures b, d, f) with different ratios: $R_{g v}=2: 3$ (subfigures a, b), $4: 5$ (subfigures c, d) , $1: 1$ (subfigures e, f). Other parameters are fixed: $R e=100, \theta=\pi / 4, V_{v}=0.244$. 


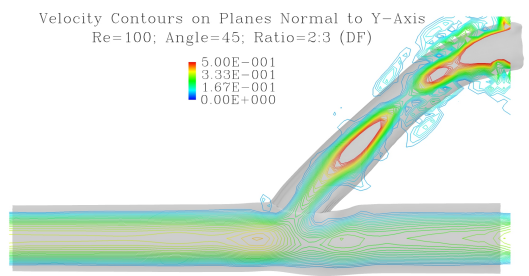

(a) Ratio $=2: 3$

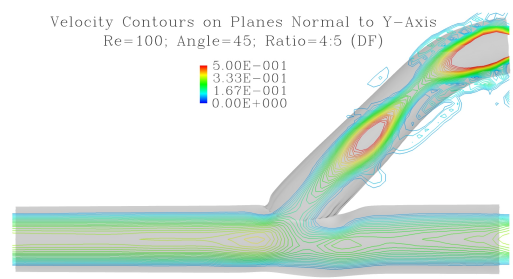

(c) Ratio $=4: 5$

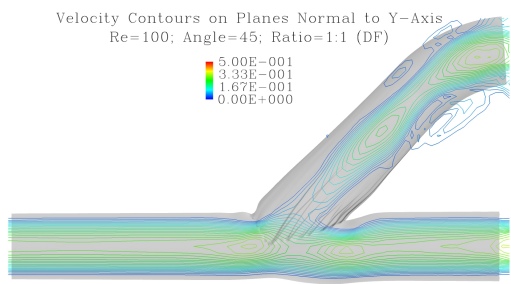

(e) Ratio=1:1

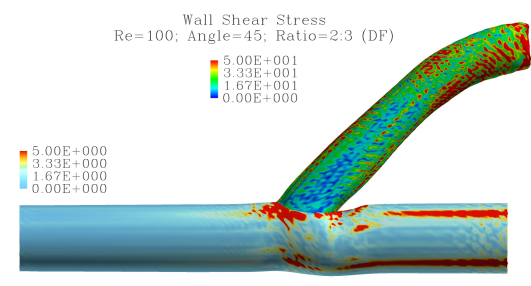

(b) Ratio $=2: 3$

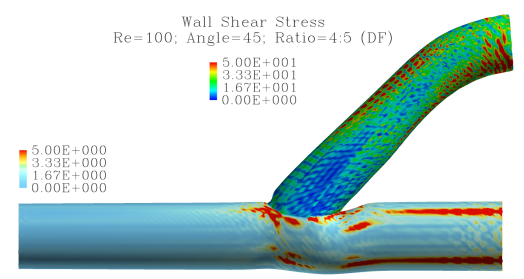

(d) Ratio= $4: 5$

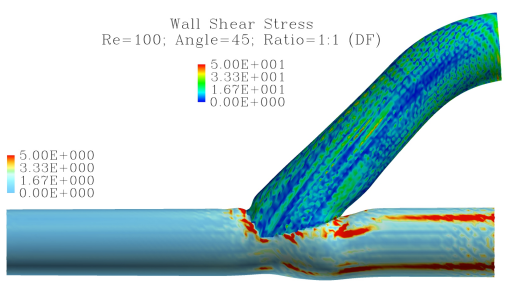

(f) Ratio=1:1

Figure 8: Effect of graft-vein diameter ratio (fixed graft influx). Velocity contours (subfigures a, c, e) on a plane $\perp$ Y-Axis and distributions of WSS (subfigures b, d, f) with different ratios: $R_{g v}=2: 3$ (subfigures a, b), $4: 5$ (subfigures c, d), $1: 1$ (subfigures e, f). Other parameters are fixed: $R e=100, \theta=\pi / 4, V_{v}=0.174$. 


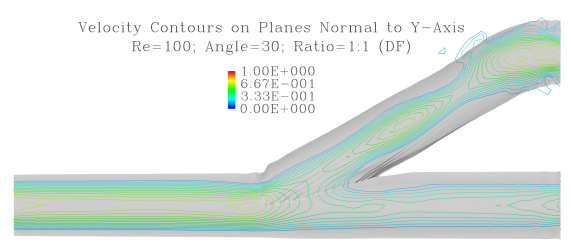

(a) Attaching Angle $=30$

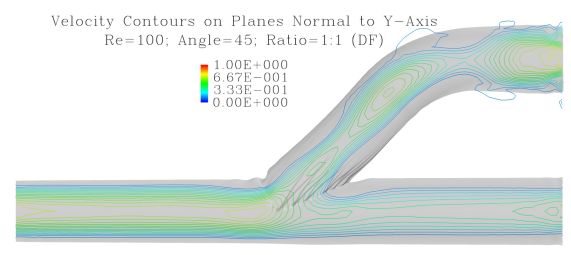

(c) Attaching Angle $=45$

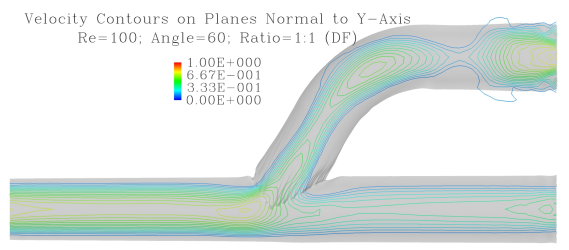

(e) Attaching Angle $=60$

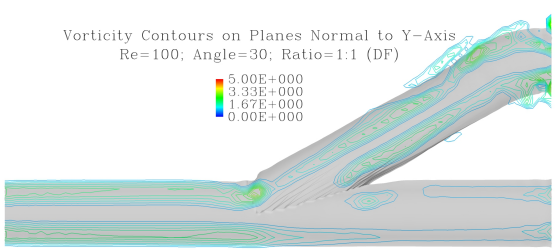

(b) Attaching Angle $=30$

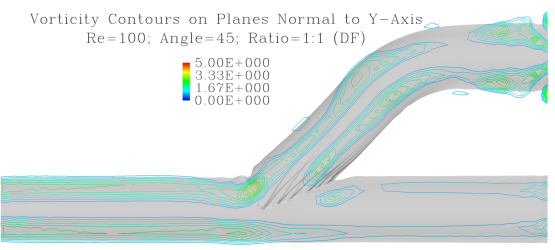

(d) Attaching Angle=45

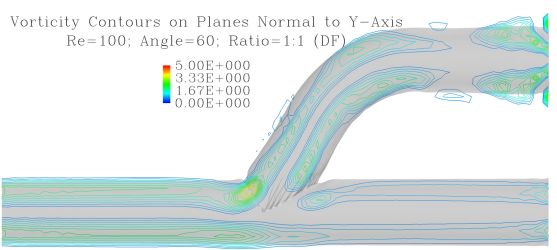

(f) Attaching Angle $=60$

Figure 9: Effect of graft attaching angle. Contours of velocity (subfigures a, c, e) and vorticity (subfigures $\mathrm{b}, \mathrm{d}, \mathrm{f}$ ) on a plane $\perp \mathrm{Y}$-Axis with different attaching angles: $\theta=\pi / 6$ (subfigures a, b), $\pi / 4$ (subfigures c, d),$\pi / 3$ (subfigures e, f). Other parameters are fixed: $R e=100, R_{g v}=$ $1: 1, V_{v}=0.244$. 


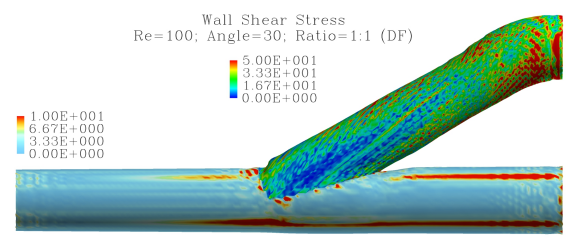

(a) Attaching Angle $=30$

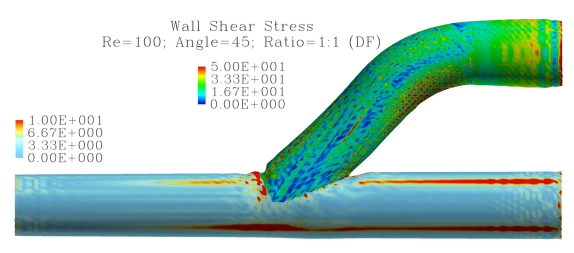

(c) Attaching Angle $=45$

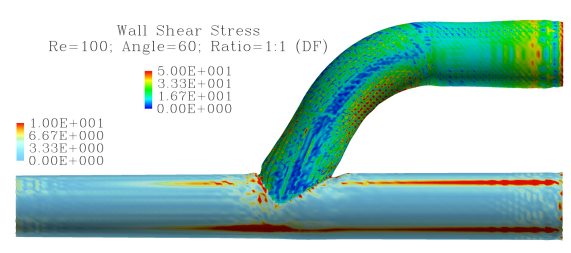

(e) Attaching Angle $=60$

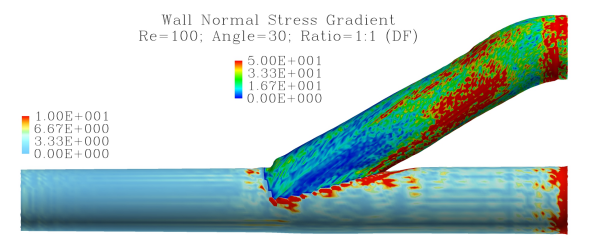

(b) Attaching Angle $=30$

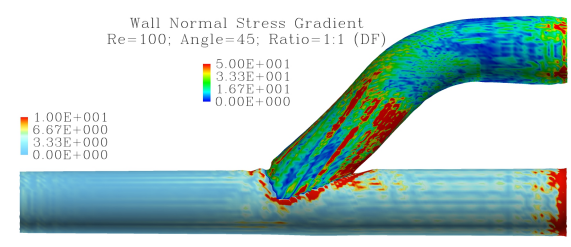

(d) Attaching Angle=45

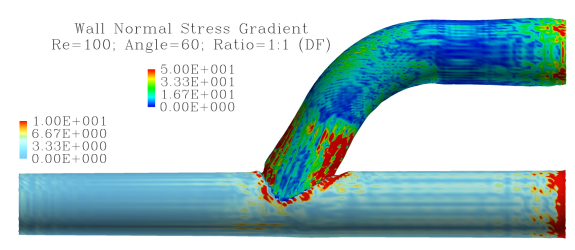

(f) Attaching Angle $=60$

Figure 10: Effect of graft attaching angle. Distributions of WSS (subfigures a, c, e) and WNSG (subfigures b, d, f) with different attaching angles: $\theta=\pi / 6$ (subfigures a, b), $\pi / 4$ (subfigures c, d),$\pi / 3$ (subfigures e, f). Other parameters are fixed: $R e=100, R_{g v}=1: 1, V_{v}=0.244$. 


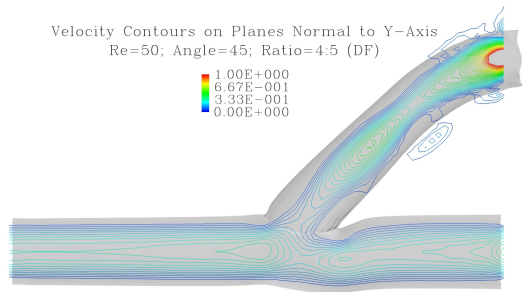

(a) $\operatorname{Re}=50$

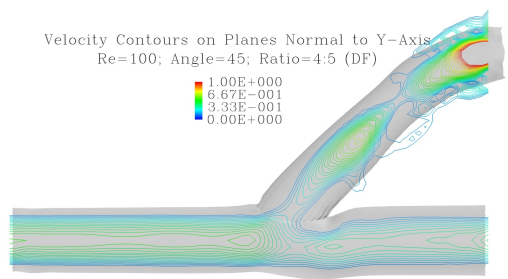

(c) $\operatorname{Re}=100$

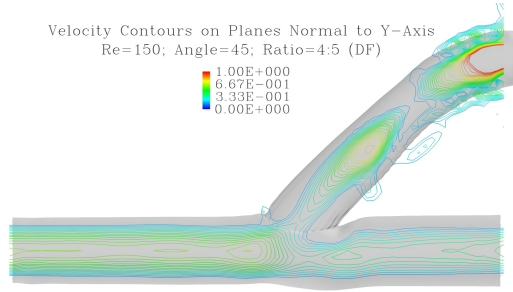

(e) $\operatorname{Re}=150$

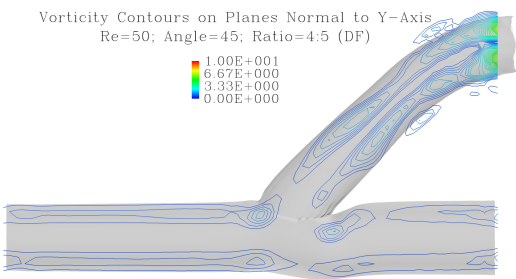

(b) $\operatorname{Re}=50$

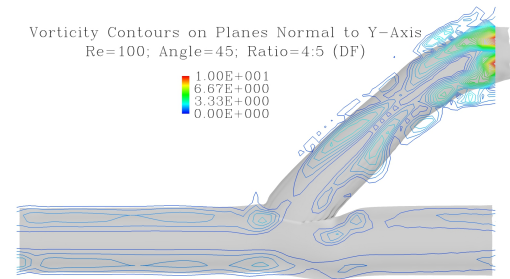

(d) $\mathrm{Re}=100$

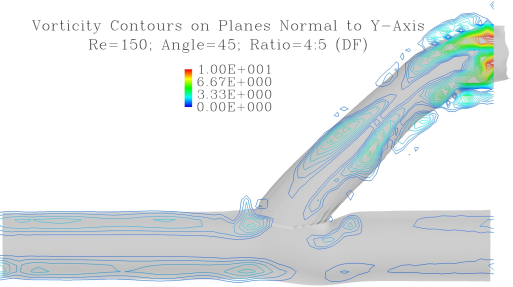

(f) $\operatorname{Re}=150$

Figure 11: Effect of flow Reynolds number. Contours of velocity (subfigures a, c, e) and vorticity (subfigures b, d, f) on a plane $\perp$ Y-Axis with different Reynolds numbers: $R e=50$ (subfigures a, b), 100 (subfigures c, d) , 150 (subfigures e, f). Other parameters are fixed: $\theta=\pi / 4, R_{g v}=4: 5, V_{v}=0.244$. 


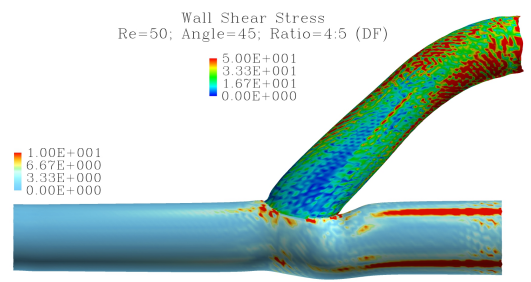

(a) $\operatorname{Re}=50$

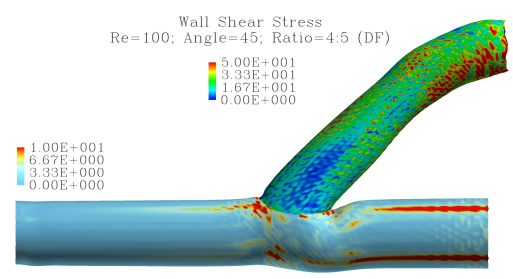

(c) $\operatorname{Re}=100$

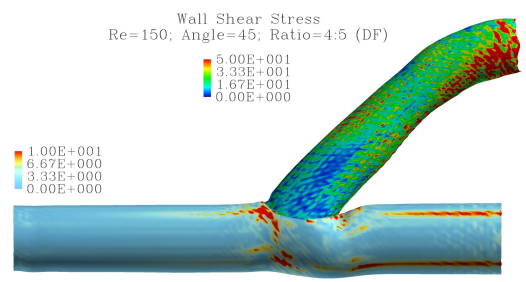

(e) $\operatorname{Re}=150$

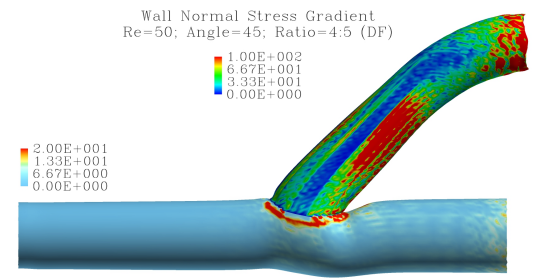

(b) $\operatorname{Re}=50$

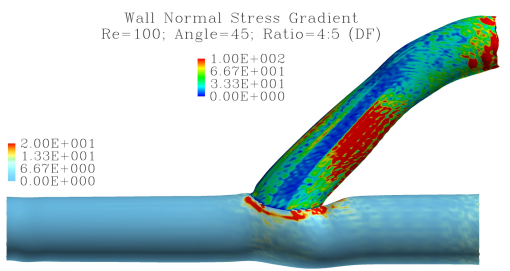

(d) $\mathrm{Re}=100$

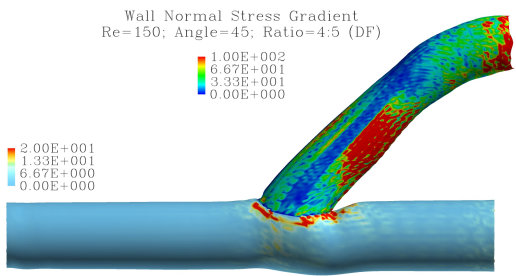

(f) $\mathrm{Re}=150$

Figure 12: Effect of flow Reynolds number. Distributions of WSS (subfigures a, c, e) and WNSG (subfigures b, d, f) with different Reynolds numbers: $R e=50$ (subfigures a, b), 100 (subfigures c, d) , 150 (subfigures e, f). Other parameters are fixed: $\theta=\pi / 4, R_{g v}=4$ : $5, V_{v}=0.244$ 


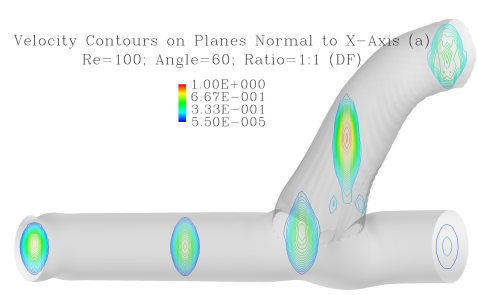

(a) Low Speed Case

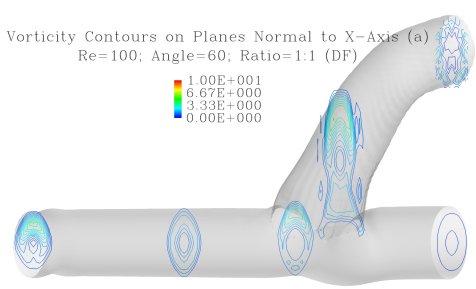

(c) Low Speed Case

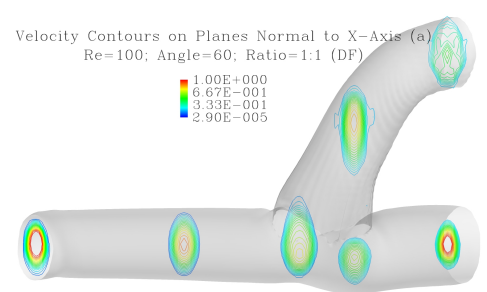

(b) High Speed Case

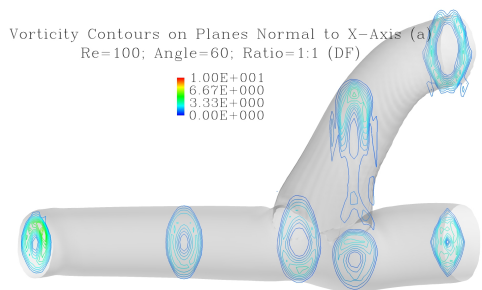

(d) High Speed Case

Figure 13: Effect of vein-inlet flow speed. Contours of velocity (subfigures a, b) and vorticity (subfigures c, d) on planes $\perp \mathrm{X}$-Axis with different vein-inlet flow speed: $V_{v}=0.1$ (subfigures a, c), 0.6 (subfigures b, d). Other parameters are fixed: $R e=100, \theta=\pi / 3, R_{g v}=1: 1$. 


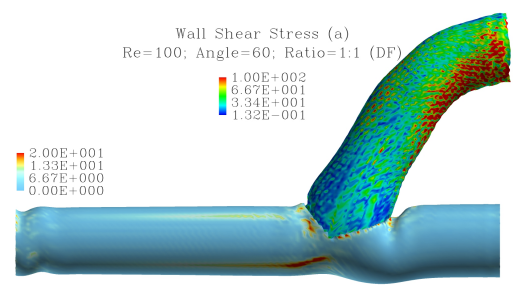

(a) Low Speed Case

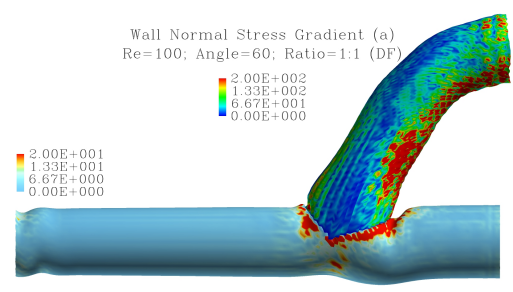

(c) Low Speed Case

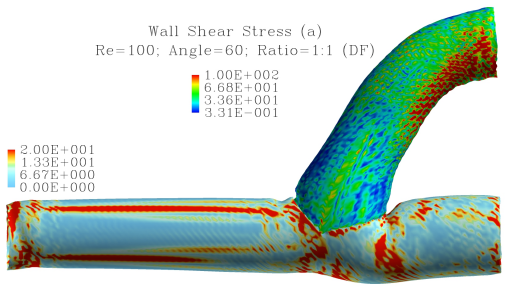

(b) High Speed Case

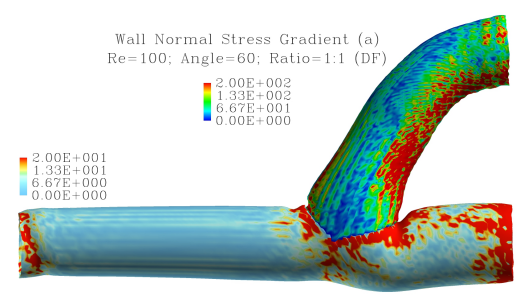

(d) High Speed Case

Figure 14: Effect of vein-inlet flow speed. Distributions of WSS (subfigures a, b) and WNSG (subfigures c, d) with different vein inlet speed: $V_{v}=0.1$ (subfigures a, c), 0.6 (subfigures b, d). Other parameters are fixed: $R e=100, \theta=\pi / 3, R_{g v}=1: 1$. 


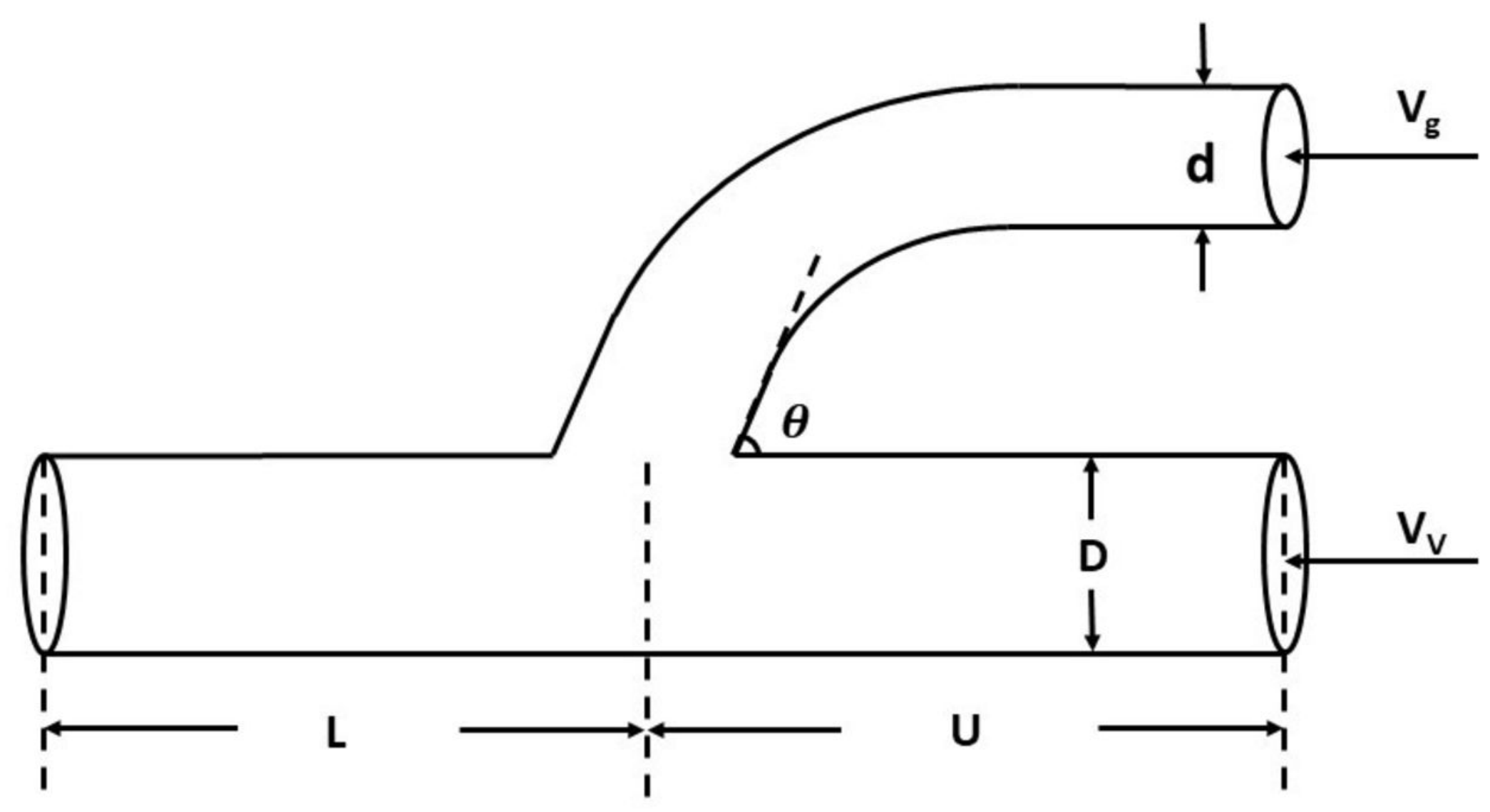




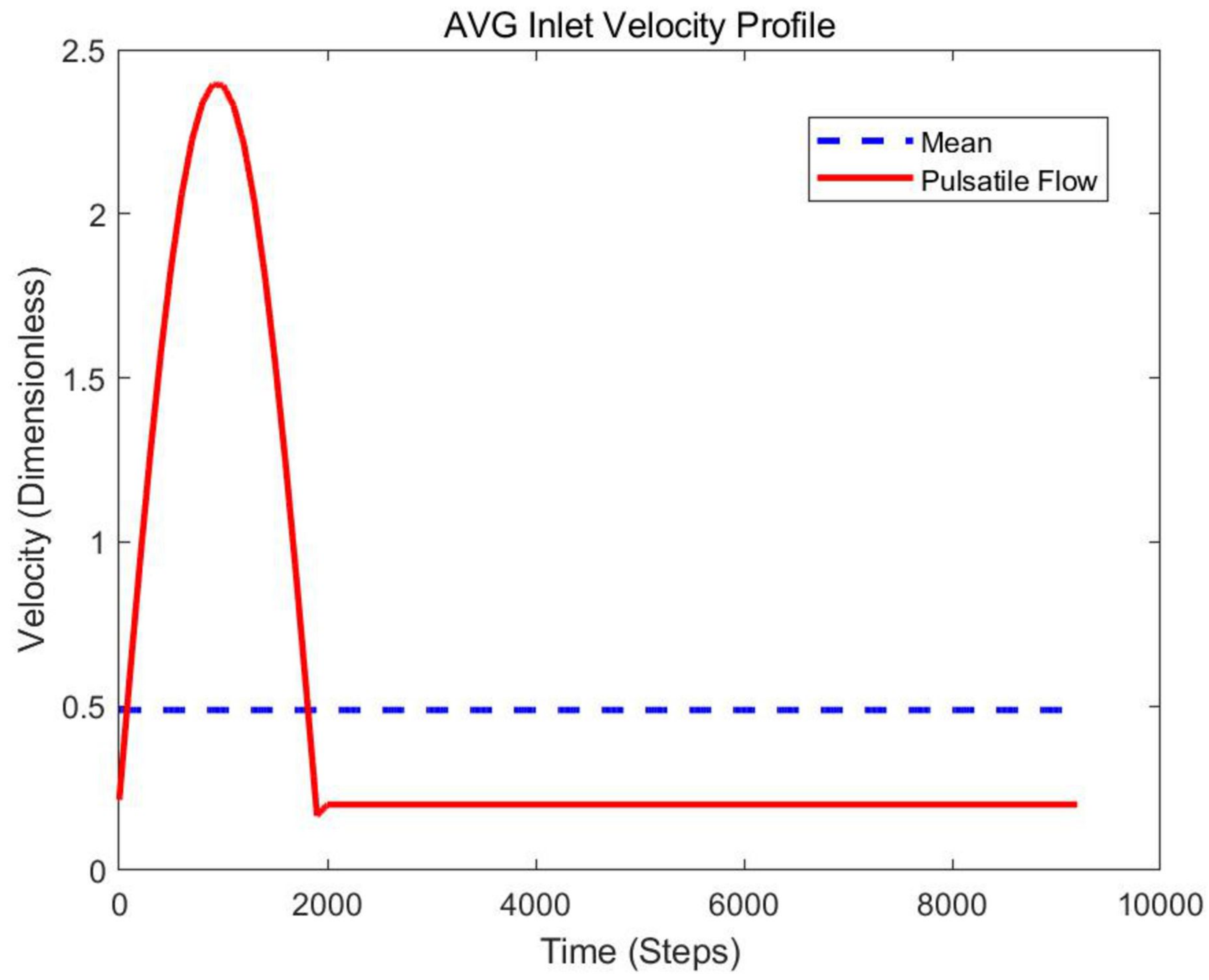




$$
\infty
$$


<smiles>[CH]=C</smiles> 


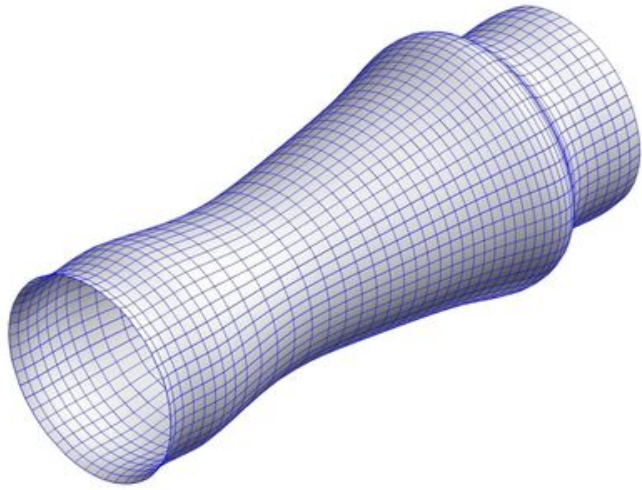




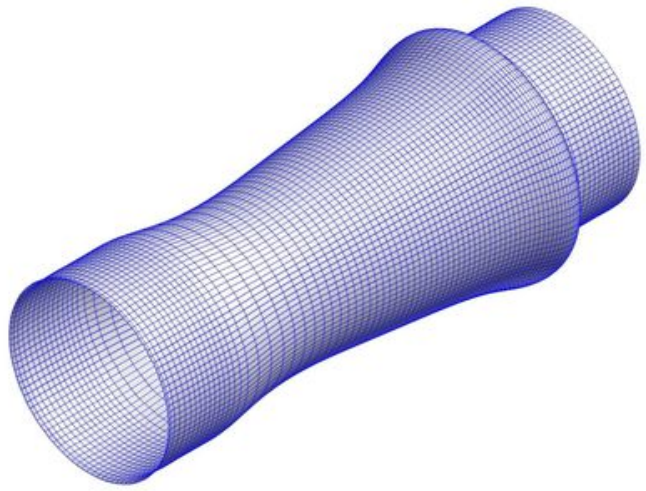




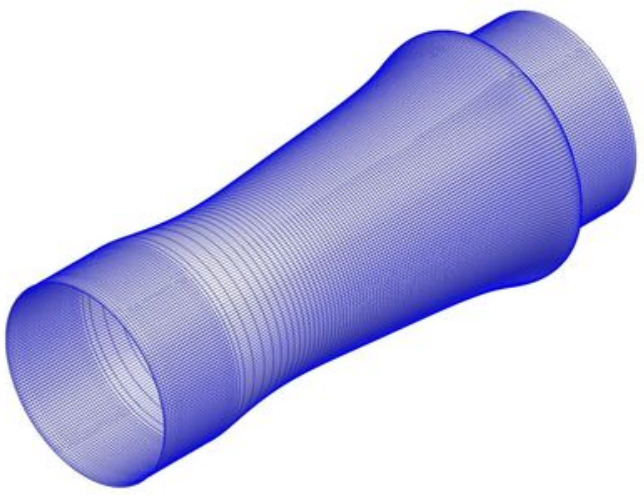




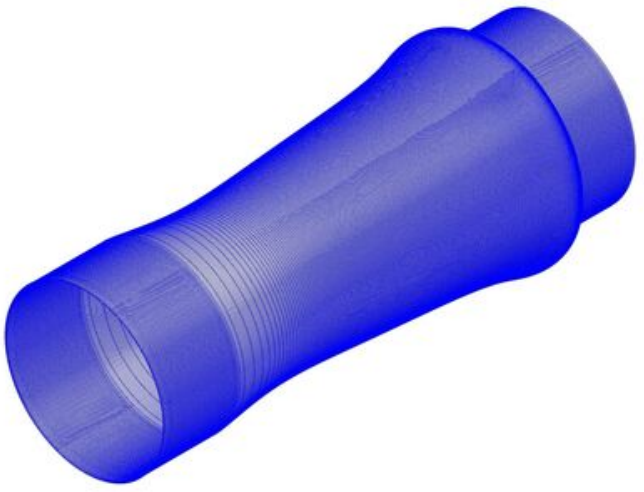




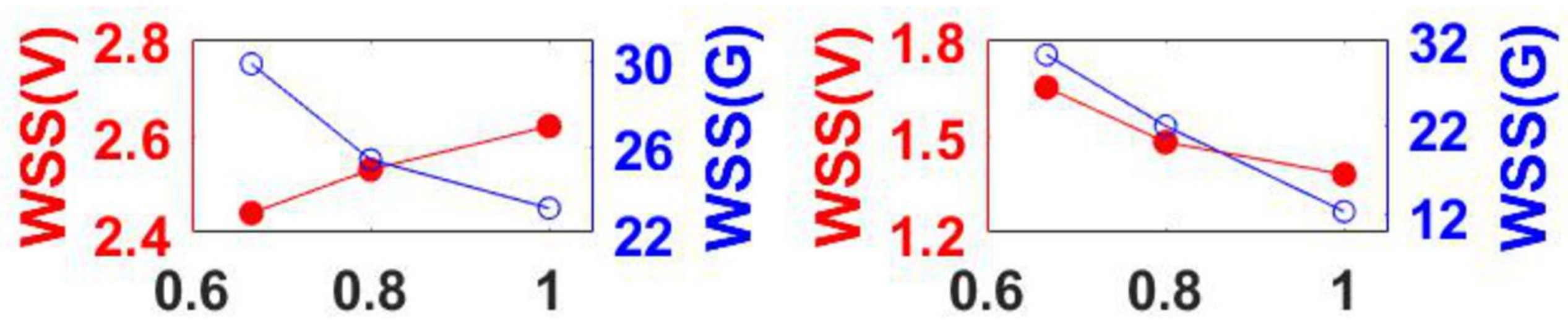

Ratio
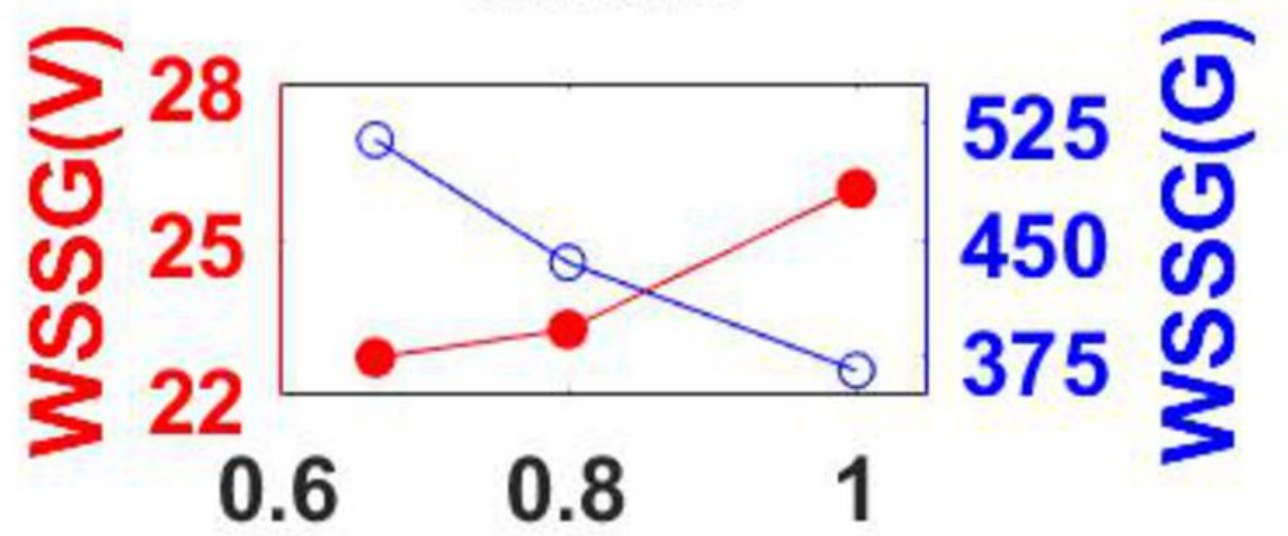

Ratio

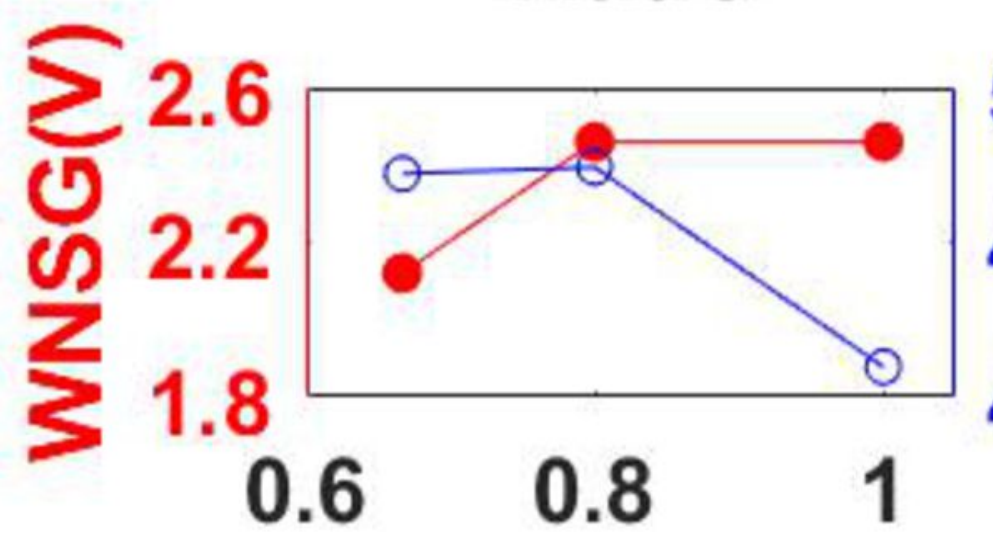

Ratio

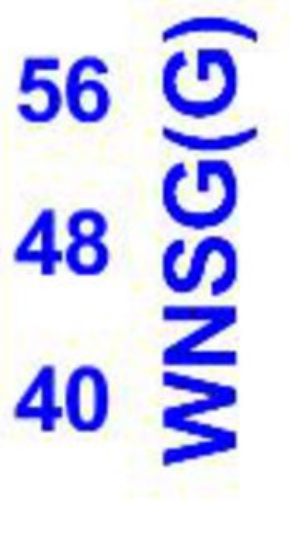

\section{Ratio}

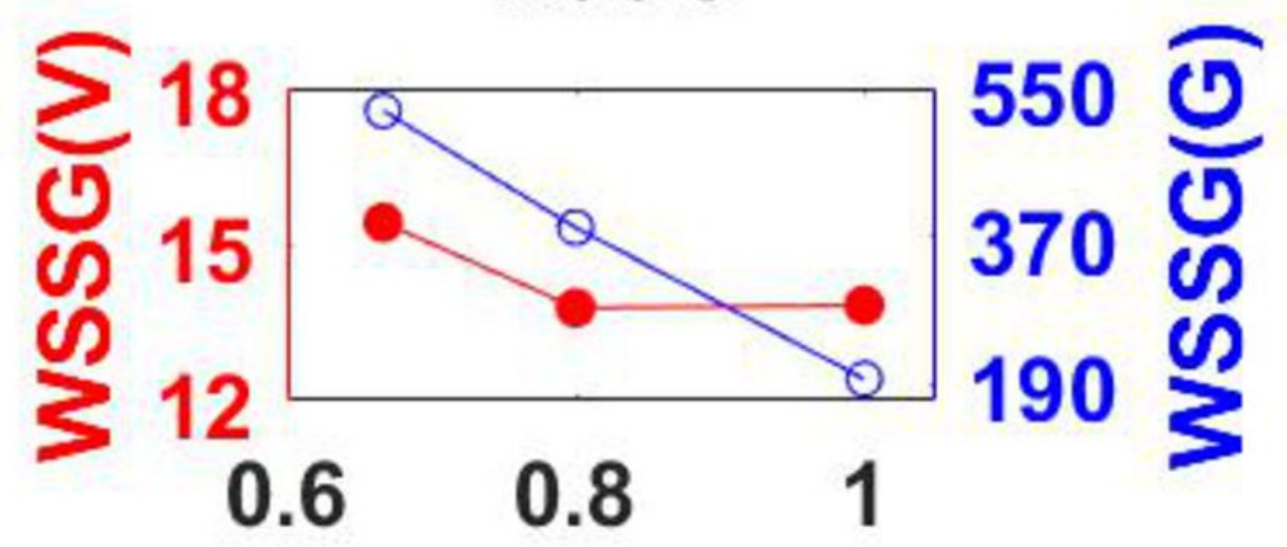

Ratio

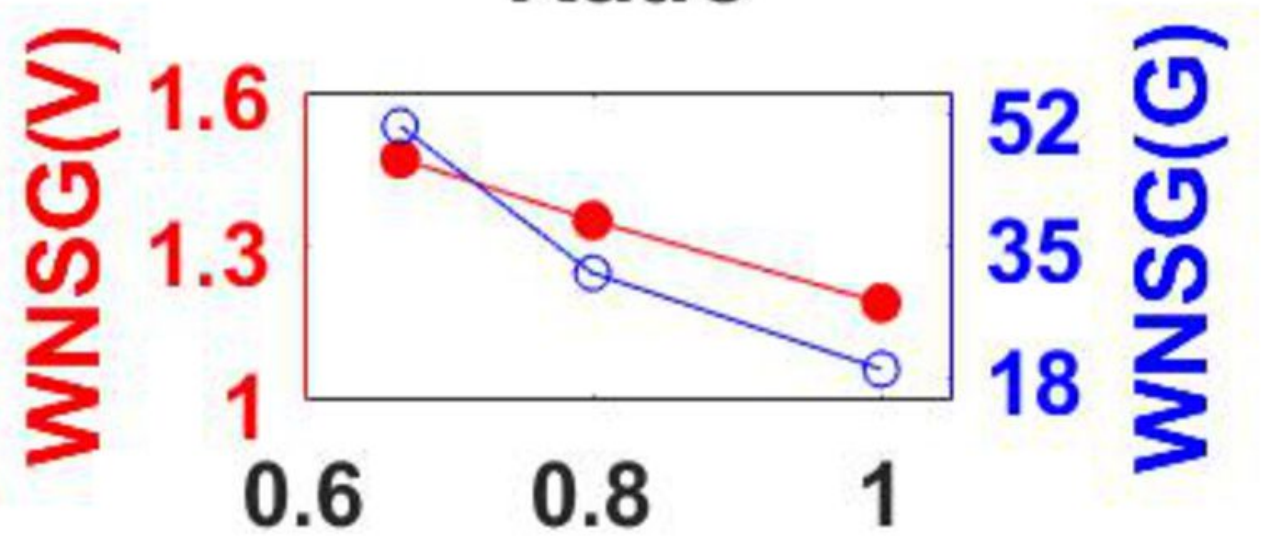

Ratio 

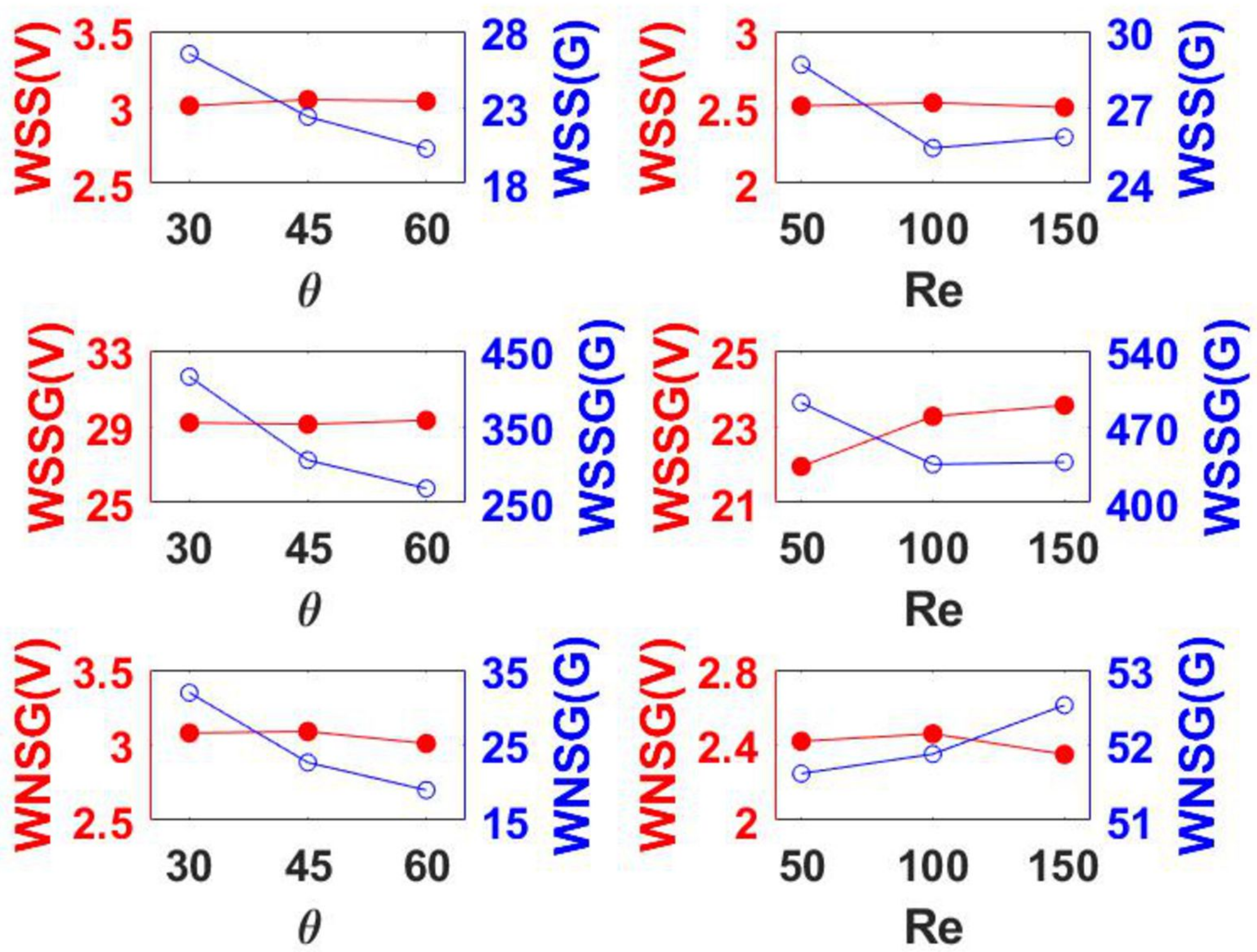

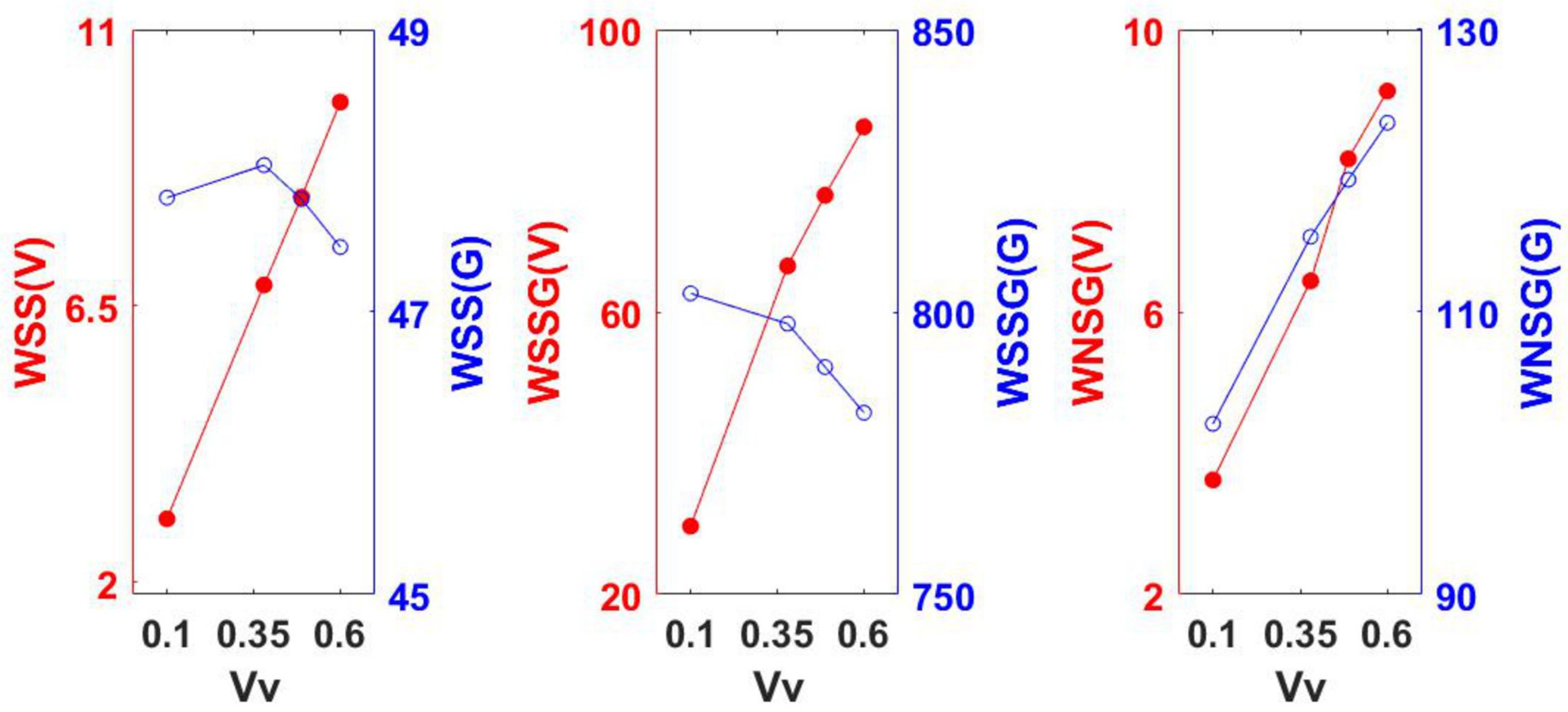
Velocity Contours on Planes Normal to Y-Axis Re=100; Angle=45; Ratio=2:3 (DF)

$1.00 \mathrm{E}+000$
$6.67 \mathrm{E}-001$
$3.33 \mathrm{E}-001$
$0.00 \mathrm{E}+000$ 
Vorticity Contours on Planes Normal to Y-Axis Re=100; Angle=45; Ratio=2:3 (DF)

1.00E+001

$6.67 \mathrm{E}+000$

$3.33 \mathrm{E}+000$

$0.00 \mathrm{E}+000$
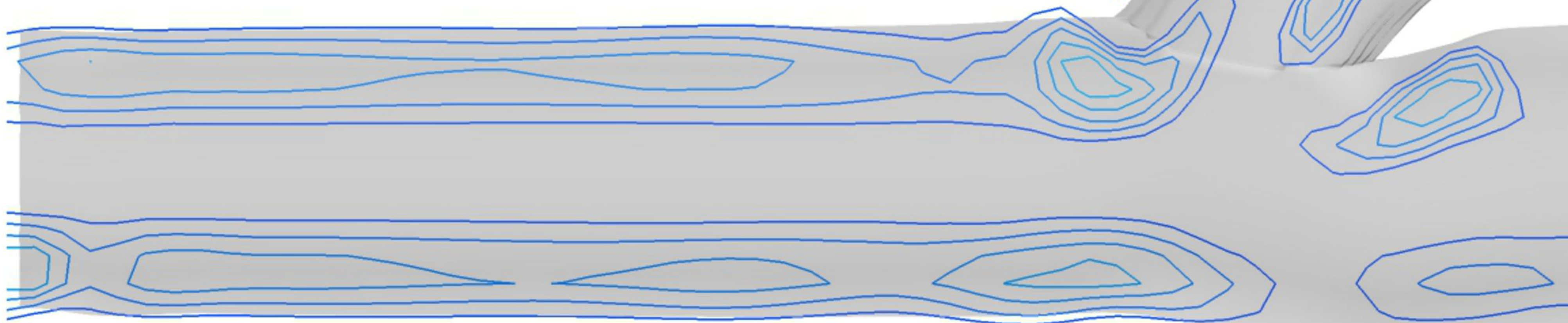
Velocity Contours on Planes Normal to Y-Axis

$$
\begin{aligned}
& \text { Re=100; Angle=45; Ratio=4:5 (DF) } \\
& \text { - } 1.00 \mathrm{E}+000 \\
& 6.67 \mathrm{E}-001 \\
& 3.33 \mathrm{E}-001 \\
& 0.00 \mathrm{E}+000
\end{aligned}
$$


Vorticity Contours on Planes Normal to Y-Axis

$$
\begin{gathered}
\text { Re=100; Angle=45; Ratio=4:5 (DF) } \\
\qquad \begin{array}{c}
1.00 \mathrm{E}+001 \\
6.67 \mathrm{E}+000 \\
3.33 \mathrm{E}+000 \\
0.00 \mathrm{E}+000
\end{array}
\end{gathered}
$$

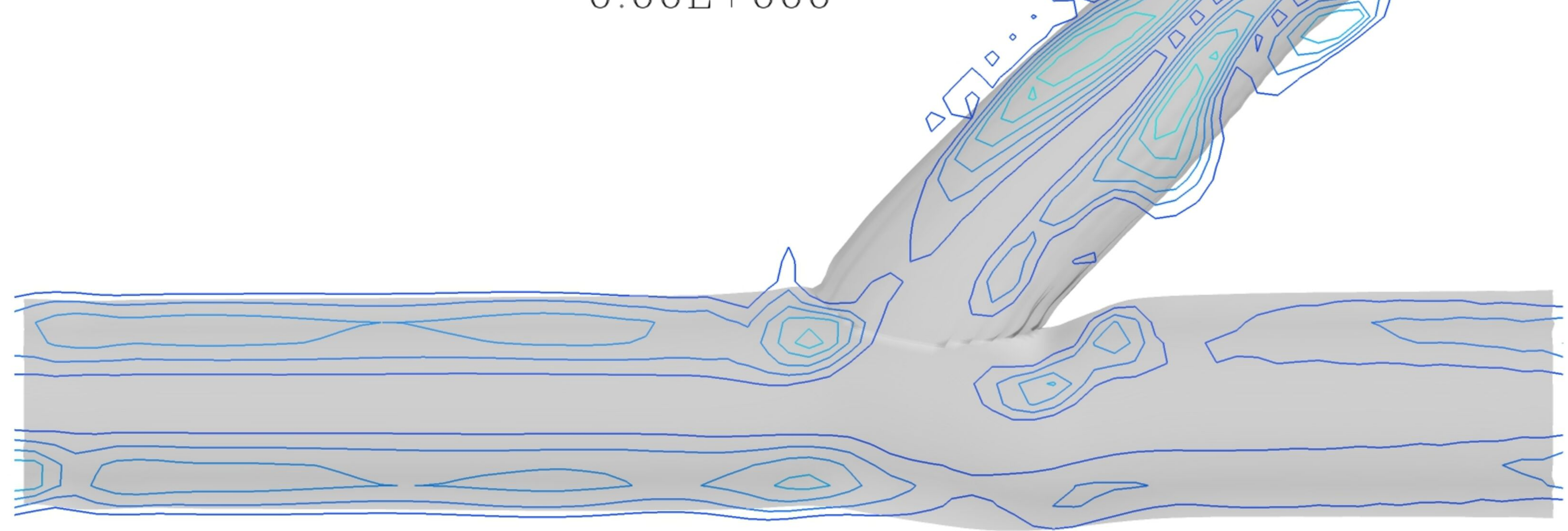


Velocity Contours on Planes Normal to Y-Axis

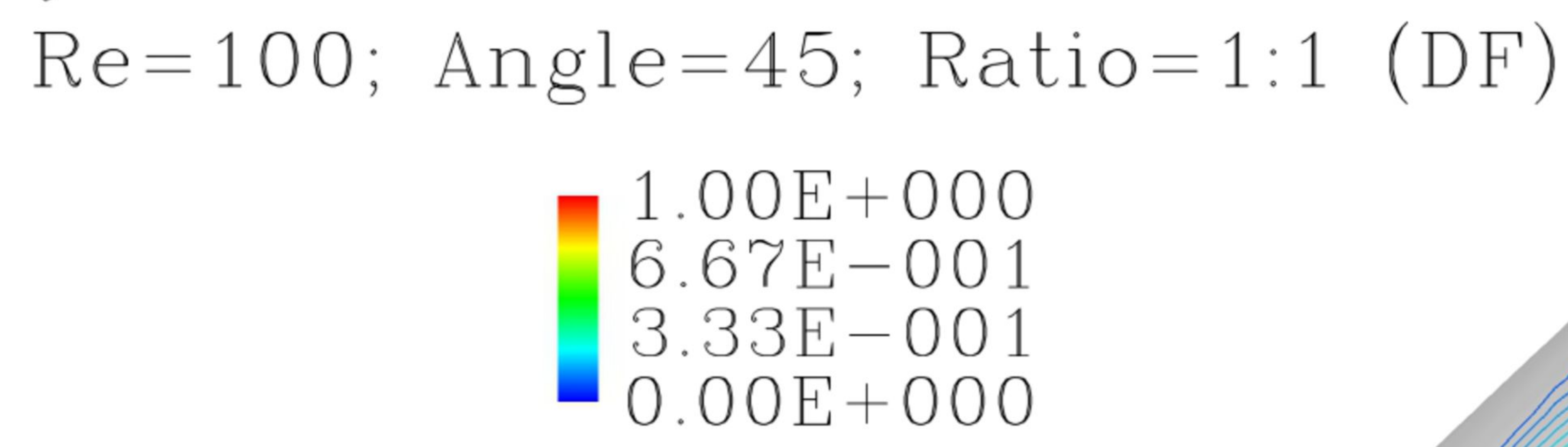


Vorticity Contours on Planes Normal to Y-Axis Re=100; Angle=45; Ratio=1:1 (DF)
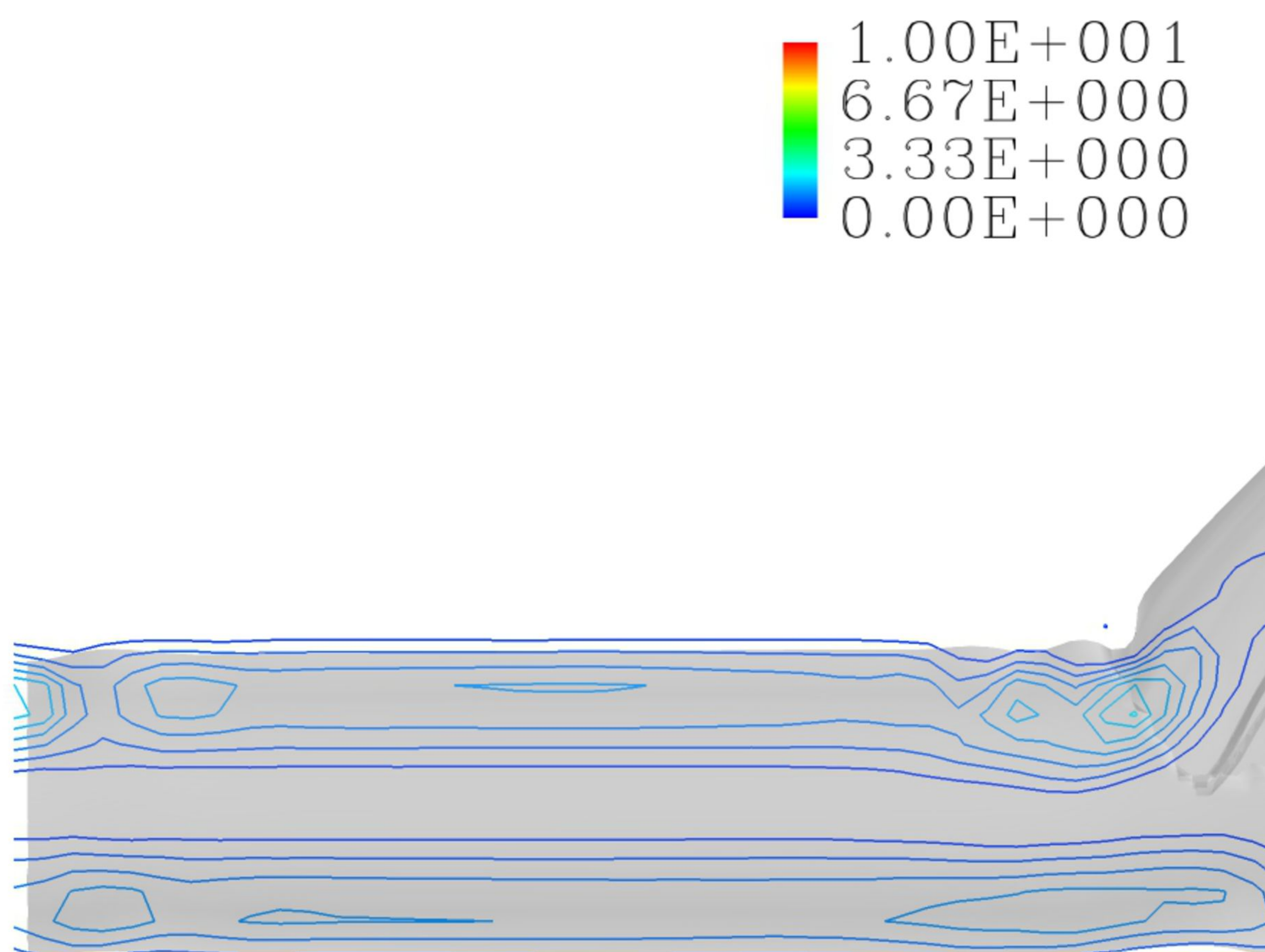
Wall Shear Stress

$$
\text { Re=100; Angle=45; Ratio=2:3 (DF) }
$$

- $1.00 \mathrm{E}+001$ $6.67 \mathrm{E}+000$ $3.33 \mathrm{E}+000$ $0.00 \mathrm{E}+000$

- $5.00 \mathrm{E}+001$ $3.33 \mathrm{E}+001$ $1.67 \mathrm{E}+001$ $0.00 \mathrm{E}+000$ 
Wall Normal Stress Gradient Re=100; Angle=45; Ratio=2:3 (DF)

2.00E+001

- $1.00 \mathrm{E}+002$

$6.67 \mathrm{E}+001$ $3.33 \mathrm{E}+001$

$0.00 \mathrm{E}+000$

1.33E +001

$6.67 \mathrm{E}+000$

$0.00 \mathrm{E}+000$ 
Wall Shear Stress

Re=100; Angle=45; Ratio=4:5 (DF)

- $1.00 \mathrm{E}+001$ $6.67 \mathrm{E}+000$

$3.33 \mathrm{E}+000$

$0.00 \mathrm{E}+000$

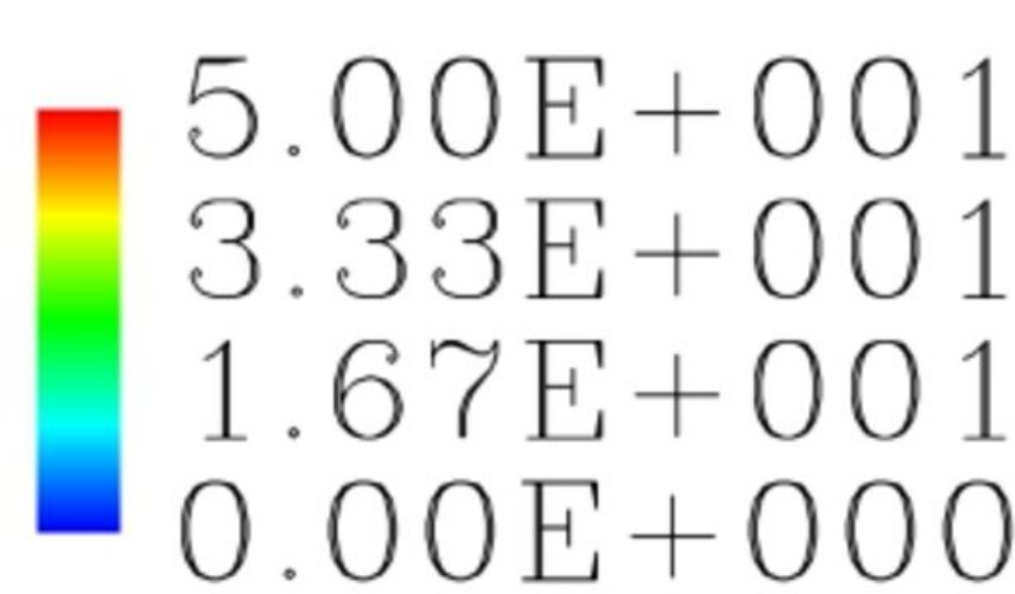


Wall Normal Stress Gradient Re=100; Angle=45; Ratio=4:5 (DF)

2.00E+001 $1.33 \mathrm{E}+001$ $6.67 \mathrm{E}+000$

$0.00 \mathrm{E}+000$ 
Wall Shear Stress

Re=100; Angle=45; Ratio=1:1 (DF)

1.00E +001 $6.67 \mathrm{E}+000$

$3.33 \mathrm{E}+000$

$0.00 \mathrm{E}+000$

- 5.00E+001

$3.33 \mathrm{E}+001$

$1.67 \mathrm{E}+001$

$0.00 \mathrm{E}+000$

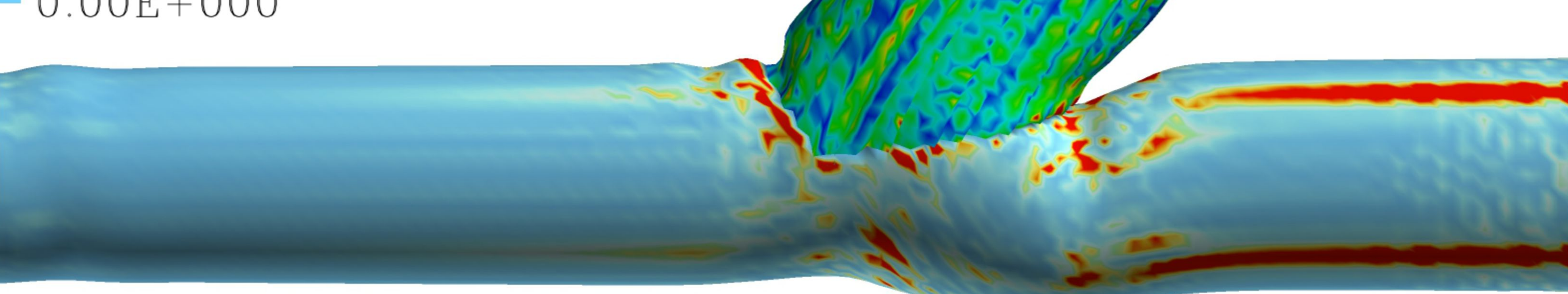


Wall Normal Stress Gradient Re=100; Angle=45; Ratio=1:1 (DF)

-2.00E+001

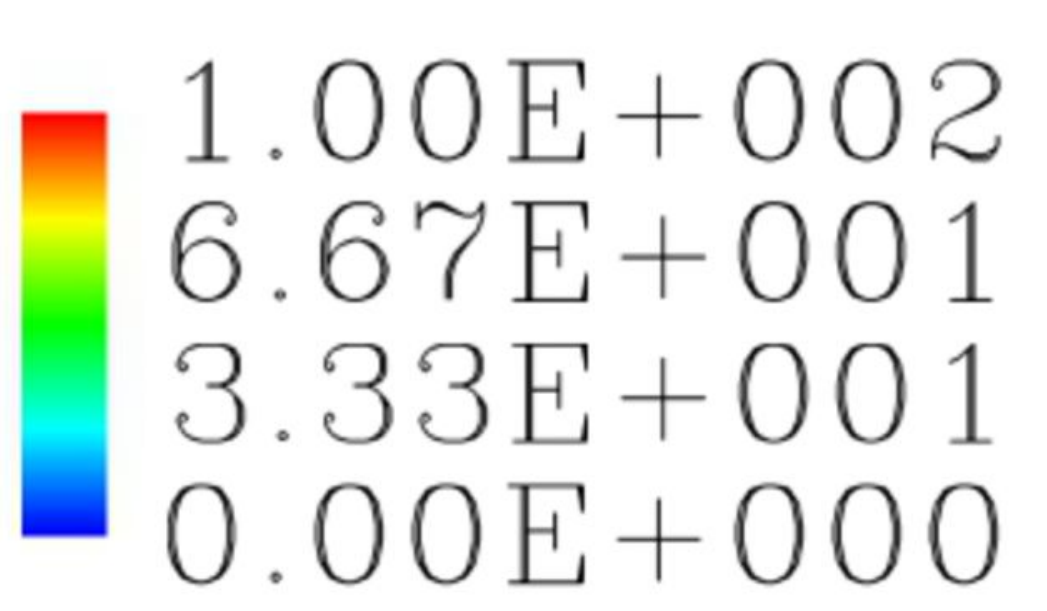

1.33E+001

$6.67 \mathrm{E}+000$

$0.00 \mathrm{E}+000$ 
Velocity Contours on Planes Normal to Y-Axis Re=100; Angle=45; Ratio=2:3 (DF)

- 5.00E-001

$3.33 \mathrm{E}-001$

$1.67 \mathrm{E}-001$

- $0.00 \mathrm{E}+000$ 
Wall Shear Stress

$$
\text { Re=100; Angle=45; Ratio=2:3 (DF) }
$$

- 5.00E+000 $3.33 \mathrm{E}+000$

$1.67 \mathrm{E}+000$

$0.00 \mathrm{E}+000$ 
Velocity Contours on Planes Normal to Y-Axis

$$
\text { Re=100; Angle=45; Ratio=4:5 (DF) }
$$

- 5.00E-001 $3.33 \mathrm{E}-001$

$1.67 \mathrm{E}-001$

$0.00 \mathrm{E}+000$ 
Wall Shear Stress

Re=100; Angle=45; Ratio=4:5 (DF)

- 5.00E+ 000 $3.33 \mathrm{E}+000$ $1.67 \mathrm{E}+000$ $0.00 \mathrm{E}+000$

- 5.00E+001 $3.33 \mathrm{E}+001$ $1.67 \mathrm{E}+001$ $0.00 \mathrm{E}+000$

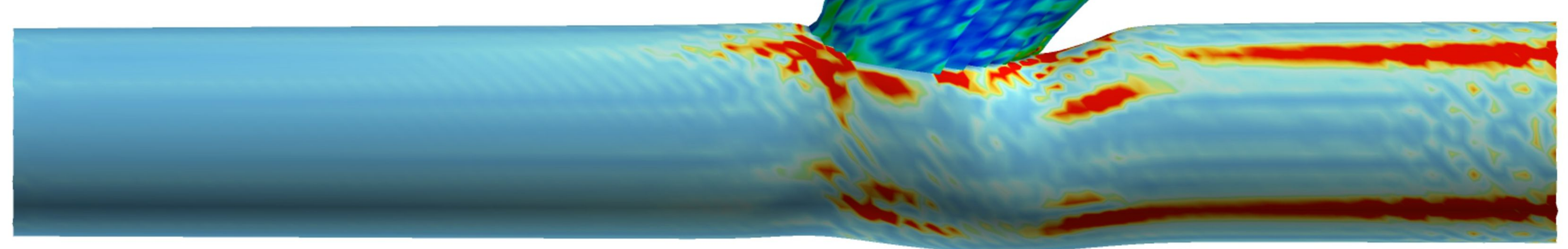


Velocity Contours on Planes Normal to Y-Axis Re=100; Angle=45; Ratio=1:1 (DF)

$5.00 \mathrm{E}-001$
$3.33 \mathrm{E}-001$
$1.67 \mathrm{E}-001$
$0.00 \mathrm{E}+000$ 
Wall Shear Stress

Re=100; Angle=45; Ratio=1:1 (DF)

5.00E+000

$3.33 \mathrm{E}+000$

$1.67 \mathrm{E}+000$

$0.00 \mathrm{E}+000$

5.00E+001

3.33E+001

1.67 $\mathrm{E}+001$

$0.00 \mathrm{E}+000$

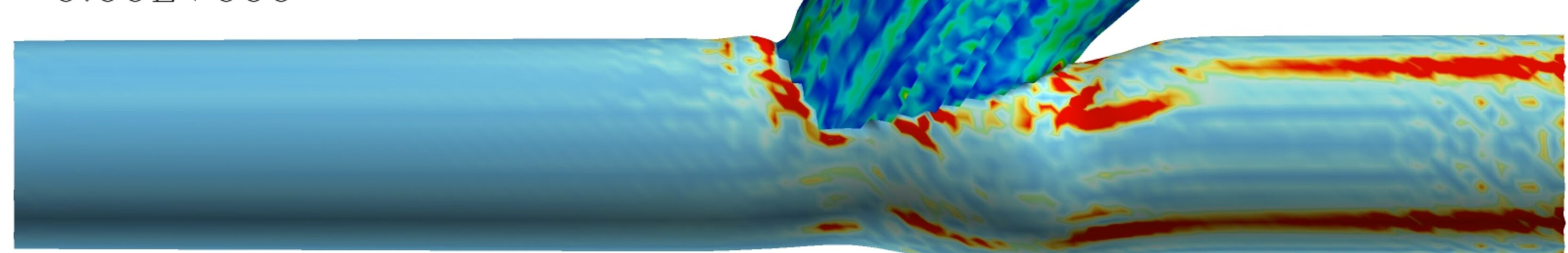


Velocity Contours on Planes Normal to Y-Axis Re=100; Angle=30; Ratio=1:1 (DF)

- 1.00E +000 $6.67 \mathrm{E}-001$ $3.33 \mathrm{E}-001$

$0.00 \mathrm{E}+000$ 
Vorticity Contours on Planes Normal to Y-Axis Re=100; Angle=30; Ratio=1:1 (DF)

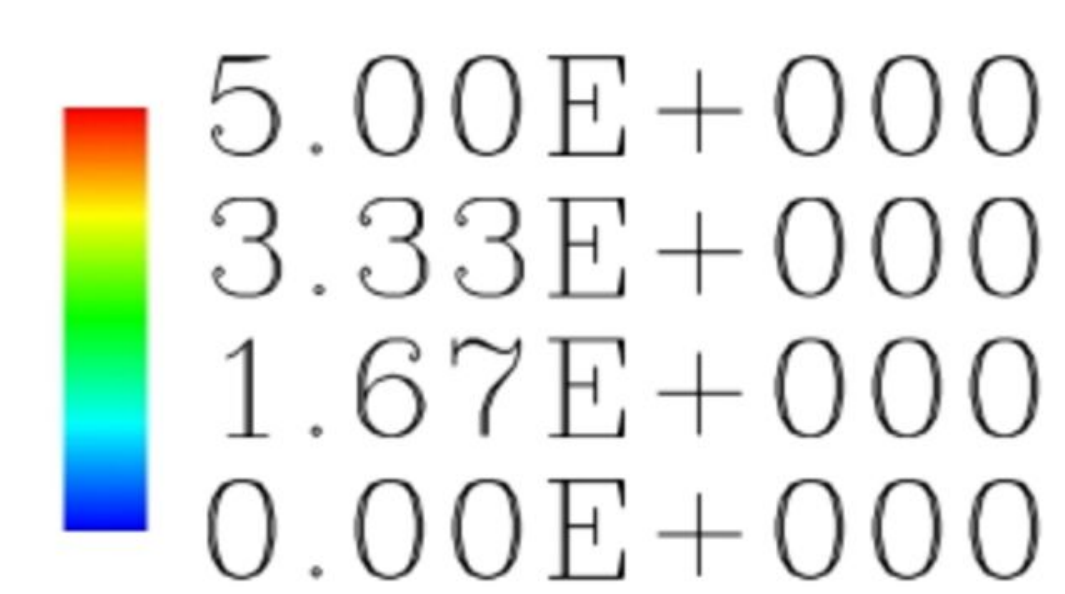


Velocity Contours on Planes Normal to Y-Axis Re=100; Angle=45; Ratio=1:1 (DF)

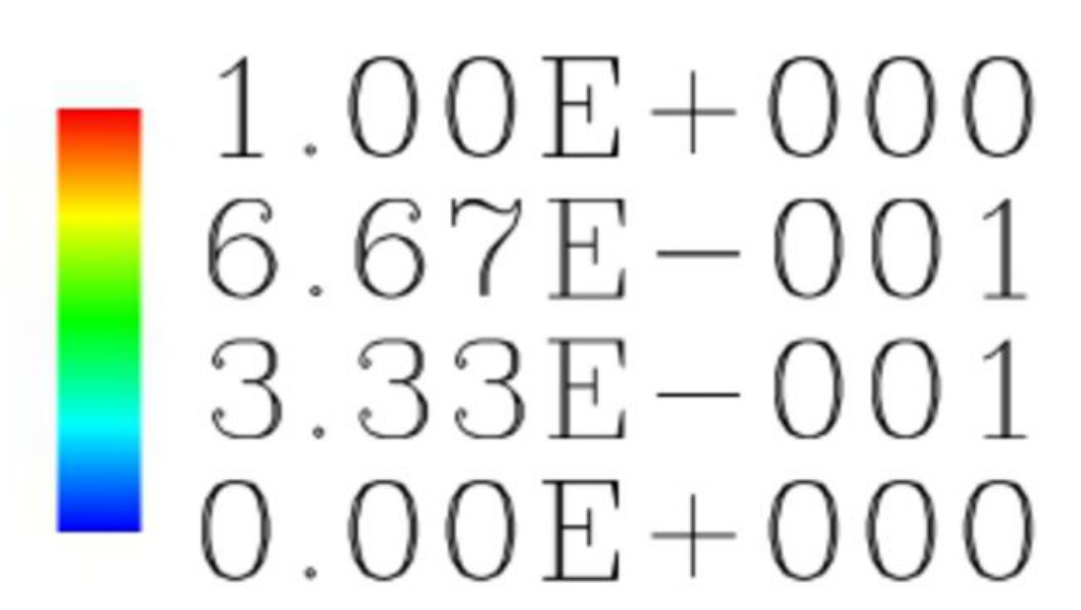


Vorticity Contours on Planes Normal to Y-Axis Re=100; Angle=45; Ratio=1:1 (DF)

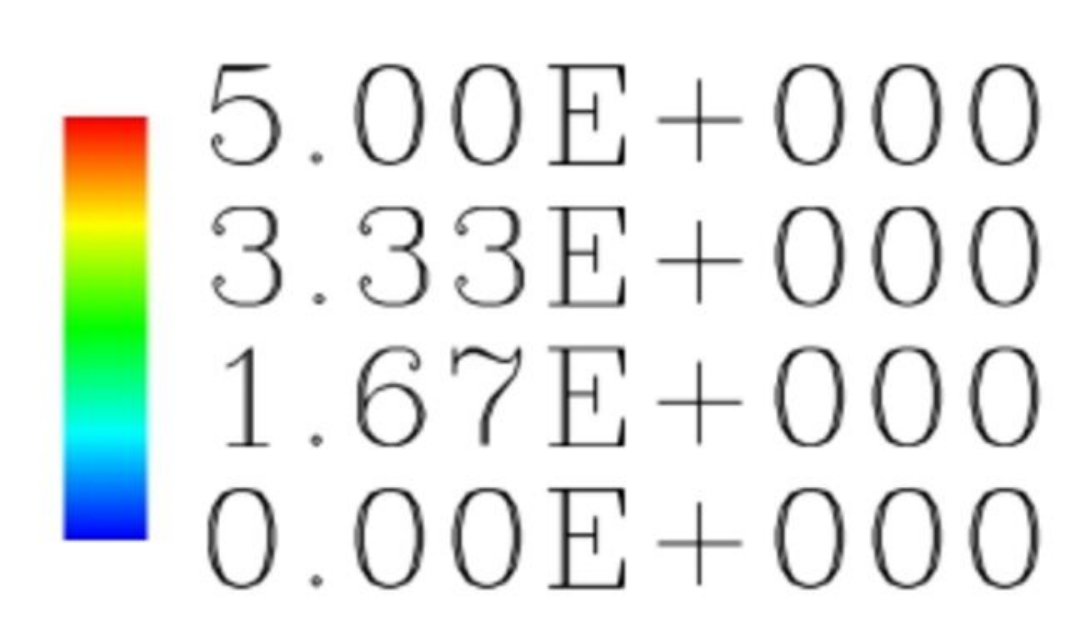


Velocity Contours on Planes Normal to Y-Axis Re=100; Angle=60; Ratio=1:1 (DF)

- $1.00 \mathrm{E}+000$

$6.67 \mathrm{E}-001$

3.33E- 001

- $0.00 \mathrm{E}+000$

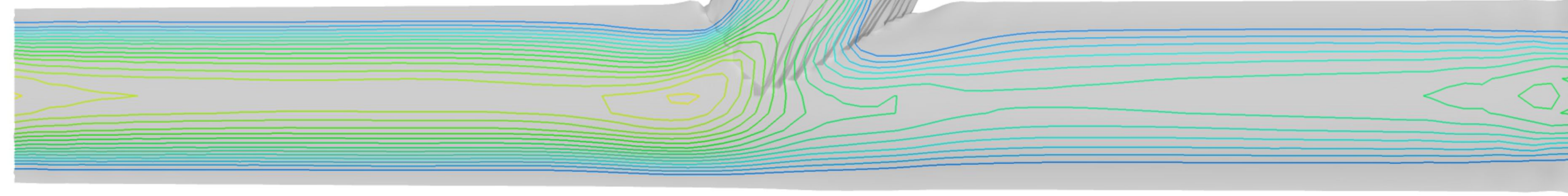


Vorticity Contours on Planes Normal to Y-Axis Re=100; Angle=60; Ratio=1:1 (DF)

5.00E +000 $3.33 \mathrm{E}+000$ $1.67 \mathrm{E}+000$ $0.00 \mathrm{E}+000$ 
Wall Shear Stress

Re=100; Angle=30; Ratio=1:1 (DF)

- $1.00 \mathrm{E}+001$

$6.67 \mathrm{E}+000$

$3.33 \mathrm{E}+000$

$0.00 \mathrm{E}+000$ 
Wall Normal Stress Gradient Re=100; Angle=30; Ratio=1:1 (DF)

- 1.00E+001 $6.67 \mathrm{E}+000$ $3.33 \mathrm{E}+000$ $0.00 \mathrm{E}+000$

- 5.00E+001 $3.33 \mathrm{E}+001$ 1.67 $\mathrm{E}+001$ $0.00 \mathrm{E}+000$

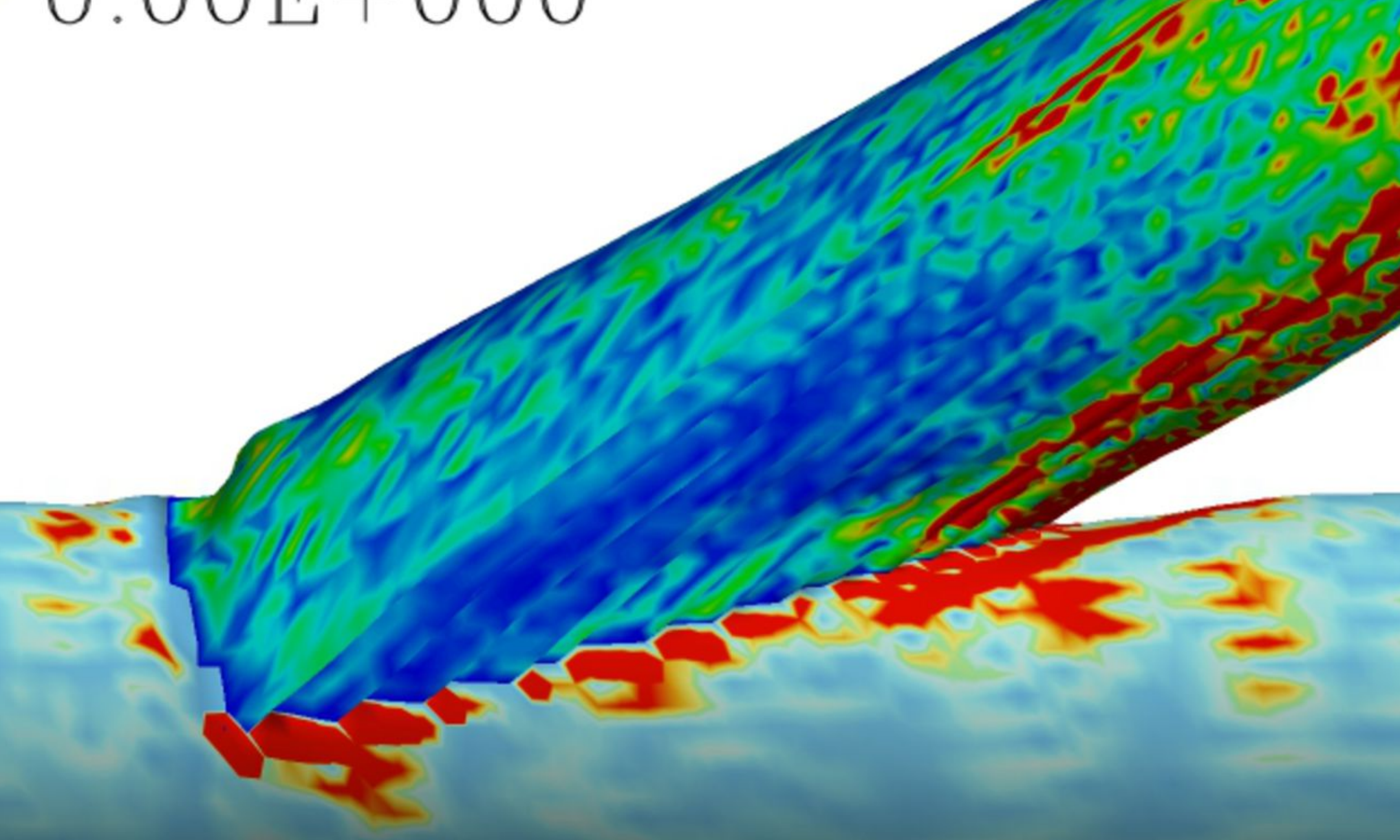


Wall Shear Stress

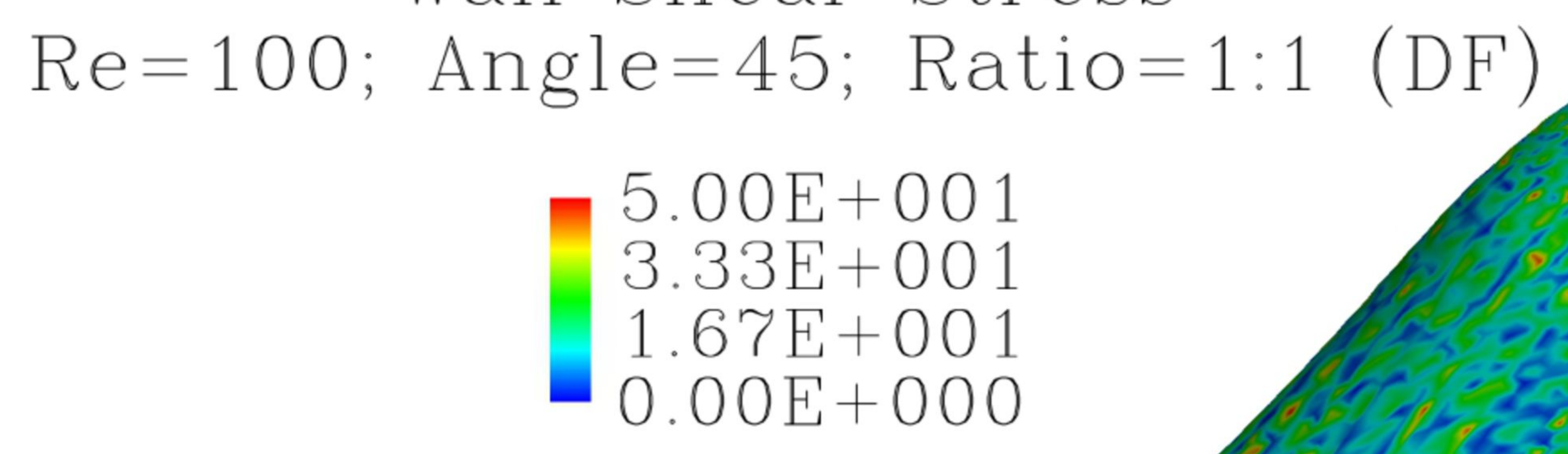

1.00E+001

$6.67 \mathrm{E}+000$

$3.33 \mathrm{E}+000$

$0.00 \mathrm{E}+000$ 
Wall Normal Stress Gradient

1.00E+001

$6.67 \mathrm{E}+000$

$3.33 \mathrm{E}+000$

$0.00 \mathrm{E}+000$ 


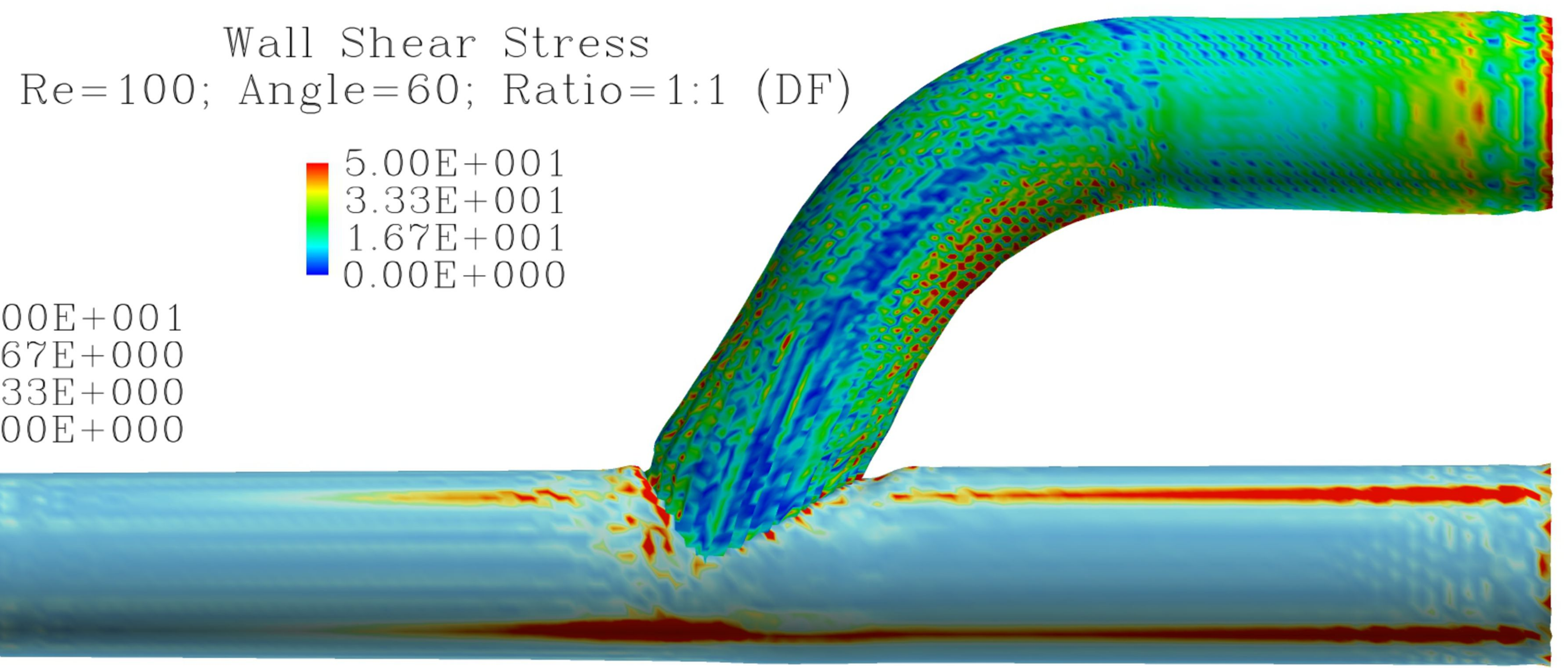


Wall Normal Stress Gradient

- $1.00 \mathrm{E}+001$ Re=100; Angle=60; Ratio=1:1 (DF)

$6.67 \mathrm{E}+000$

$3.33 \mathrm{E}+000$

$0.00 \mathrm{E}+000$

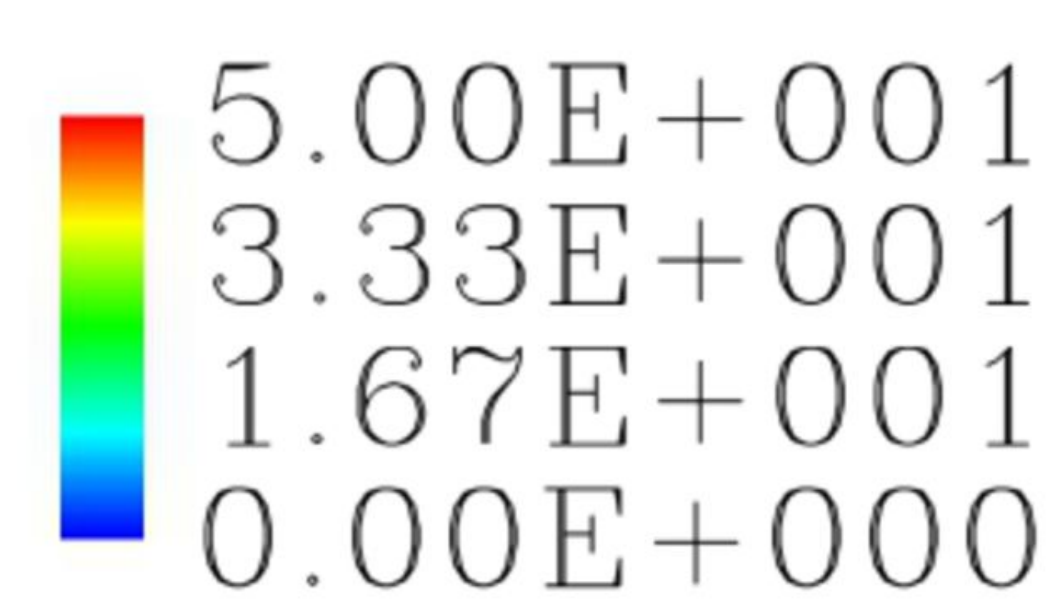


Velocity Contours on Planes Normal to Y-Axis Re=50; Angle=45; Ratio=4:5 (DF)

$1.00 \mathrm{E}+000$
$6.67 \mathrm{E}-001$
$3.33 \mathrm{E}-001$
$0.00 \mathrm{E}+000$ 
Vorticity Contours on Planes Normal to Y-Axis $\operatorname{Re}=50 ;$ Angle=45; Ratio=4:5 (DF)

$1.00 \mathrm{E}+001$
$6.67 \mathrm{E}+000$
$3.33 \mathrm{E}+000$
$0.00 \mathrm{E}+000$

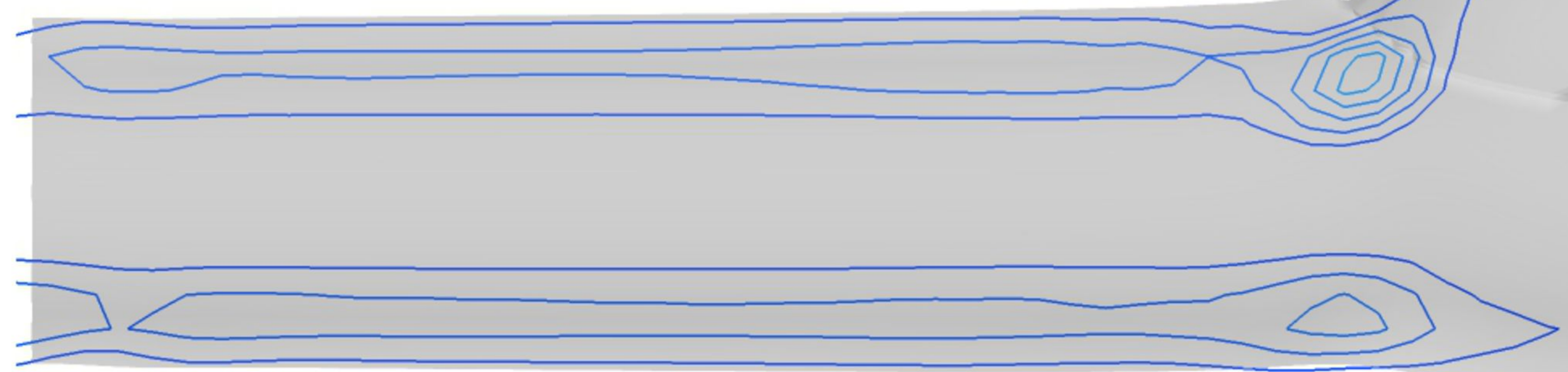


Velocity Contours on Planes Normal to Y-Axis Re=100; Angle=45; Ratio=4:5 (DF)

- $1.00 \mathrm{E}+000$

$6.67 \mathrm{E}-001$

3. 33 E-001

$0.00 \mathrm{E}+000$ 
Vorticity Contours on Planes Normal to Y-Axis

$$
\begin{gathered}
\text { Re=100; Angle=45; Ratio=4:5 (DF) } \\
\qquad \begin{array}{c}
1.00 \mathrm{E}+001 \\
6.67 \mathrm{E}+000 \\
3.33 \mathrm{E}+000 \\
0.00 \mathrm{E}+000
\end{array}
\end{gathered}
$$

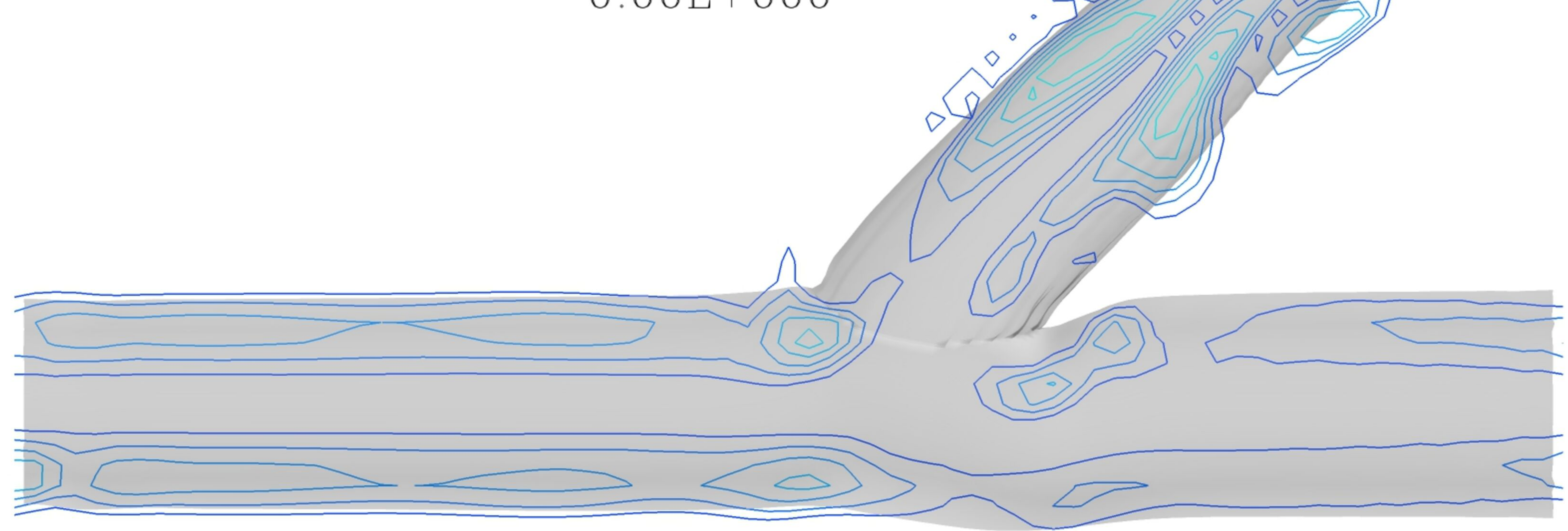


Velocity Contours on Planes Normal to Y-Axis Re=150; Angle=45; Ratio=4:5 (DF)

- $1.00 \mathrm{E}+000$ $6.67 \mathrm{E}-001$ $3.33 \mathrm{E}-001$

$0.00 \mathrm{E}+000$ 
Vorticity Contours on Planes Normal to Y-Axis Re=150; Angle=45; Ratio=4:5 (DF)

1.00E+001 $6.67 \mathrm{E}+000$

$3.33 \mathrm{E}+000$

$0.00 \mathrm{E}+000$
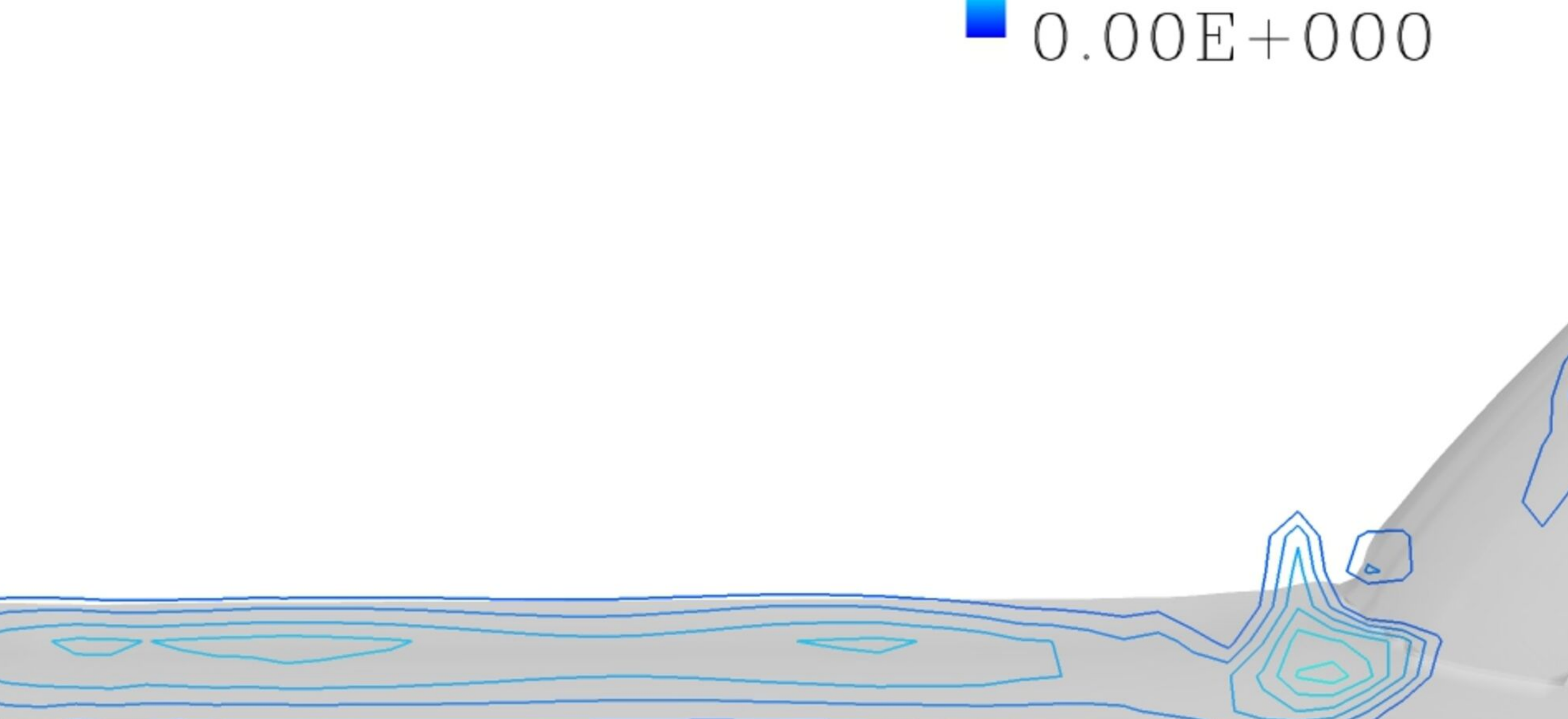
Wall Shear Stress

$$
\text { Re=50; Angle=45; Ratio=4:5 (DF) }
$$

- 1.00E+001 $6.67 \mathrm{E}+000$ $3.33 \mathrm{E}+000$ $0.00 \mathrm{E}+000$

- 5.00E+001 $3.33 \mathrm{E}+001$ $1.67 \mathrm{E}+001$ $0.00 \mathrm{E}+000$

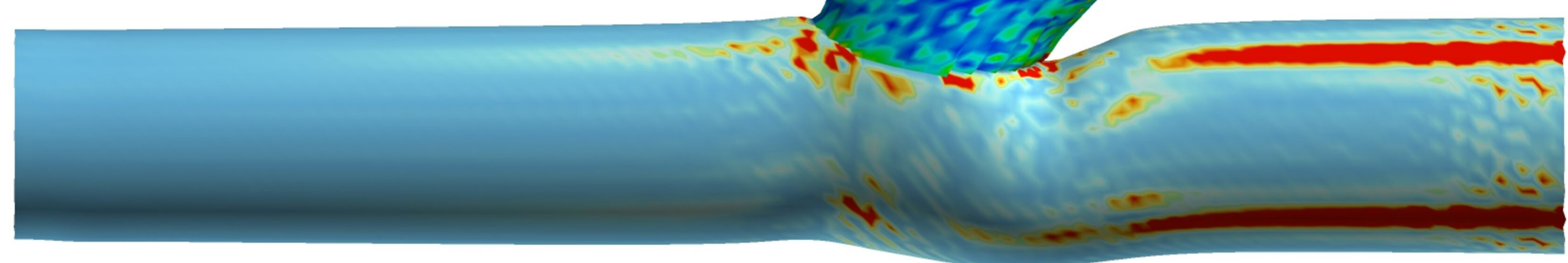


Wall Normal Stress Gradient Re=50; Angle=45; Ratio=4:5 (DF)

- 2.00E+001 $1.33 \mathrm{E}+001$ $6.67 \mathrm{E}+000$ $0.00 \mathrm{E}+000$

- 1.00E+002 $6.67 \mathrm{E}+001$ $3.33 \mathrm{E}+001$

$0.00 \mathrm{E}+000$

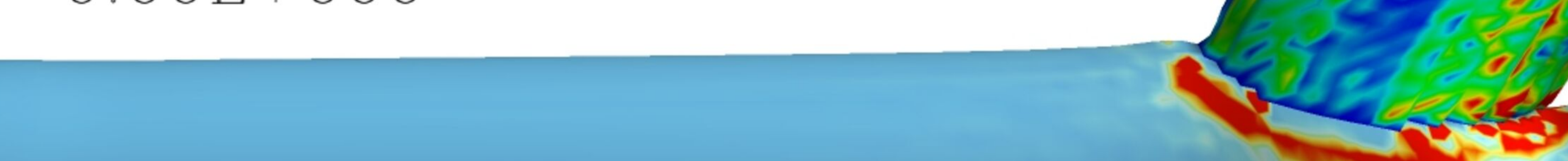


Wall Shear Stress

Re=100; Angle=45; Ratio=4:5 (DF)

$1.00 \mathrm{E}+001$

$6.67 \mathrm{E}+000$

$3.33 \mathrm{E}+000$

$0.00 \mathrm{E}+000$

- $5.00 \mathrm{E}+001$

$3.33 \mathrm{E}+001$

$1.67 \mathrm{E}+001$

- $0.00 \mathrm{E}+000$

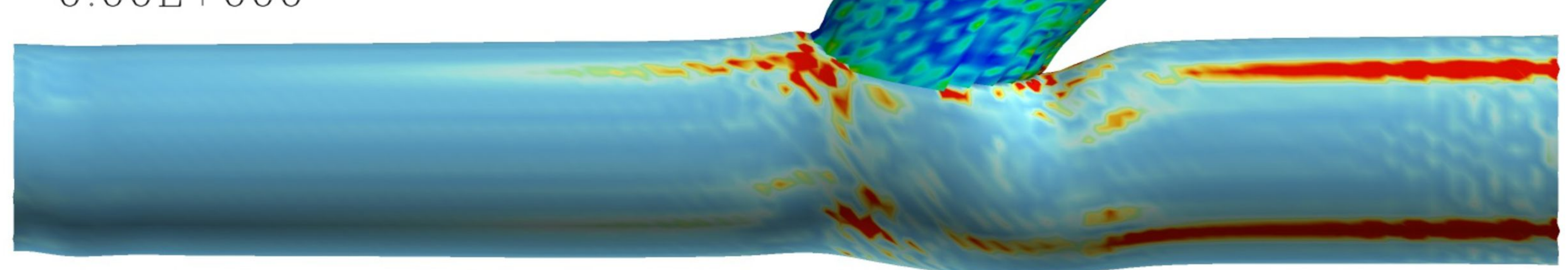


Wall Normal Stress Gradient Re=100; Angle=45; Ratio=4:5 (DF)

2.00E+001 $1.33 \mathrm{E}+001$ $6.67 \mathrm{E}+000$

$0.00 \mathrm{E}+000$ 
Wall Shear Stress

Re=150; Angle=45; Ratio=4:5 (DF)

- $1.00 \mathrm{E}+001$

$6.67 \mathrm{E}+000$

$3.33 \mathrm{E}+000$

$-0.00 \mathrm{E}+000$

- 5.00E+001

$3.33 \mathrm{E}+001$

$1.67 \mathrm{E}+001$

- $0.00 \mathrm{E}+000$

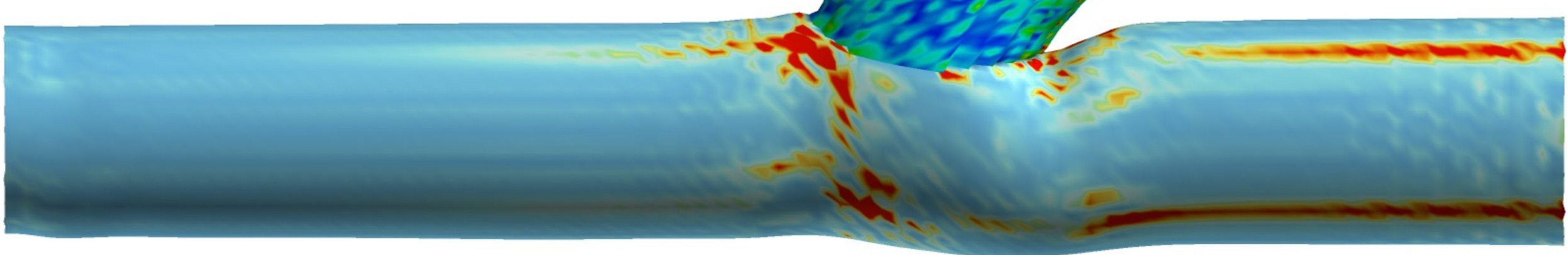


Wall Normal Stress Gradient Re=150; Angle=45; Ratio=4:5 (DF)

-2.00E+001

- $1.00 \mathrm{E}+002$ $6.67 \mathrm{E}+001$ $3.33 \mathrm{E}+001$

$0.00 \mathrm{E}+000$

$1.33 \mathrm{E}+001$

$6.67 \mathrm{E}+000$

$0.00 \mathrm{E}+000$ 
Velocity Contours on Planes Normal to X-Axis (a) Re=100; Angle=60; Ratio=1:1 (DF)

- $1.00 \mathrm{E}+000$

$6.67 \mathrm{E}-001$

3.33E-001

- $5.50 \mathrm{E}-005$ 
Velocity Contours on Planes Normal to X-Axis (a) Re=100; Angle=60; Ratio=1:1 (DF)
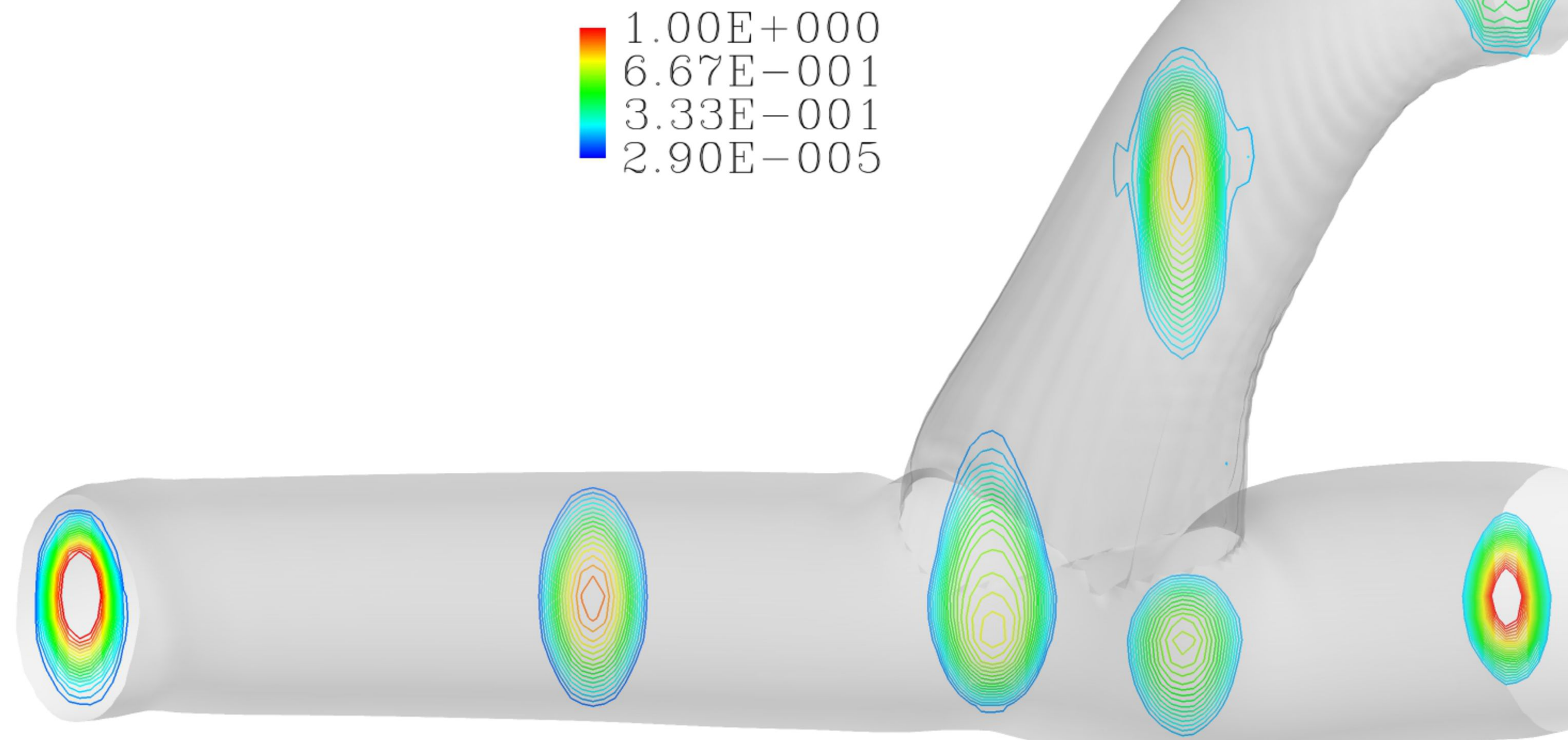
Vorticity Contours on Planes Normal to X-Axis (a) Re=100; Angle=60; Ratio=1:1 (DF)

$1.00 \mathrm{E}+001$

$6.67 \mathrm{E}+000$

$3.33 \mathrm{E}+000$

$0.00 \mathrm{E}+000$
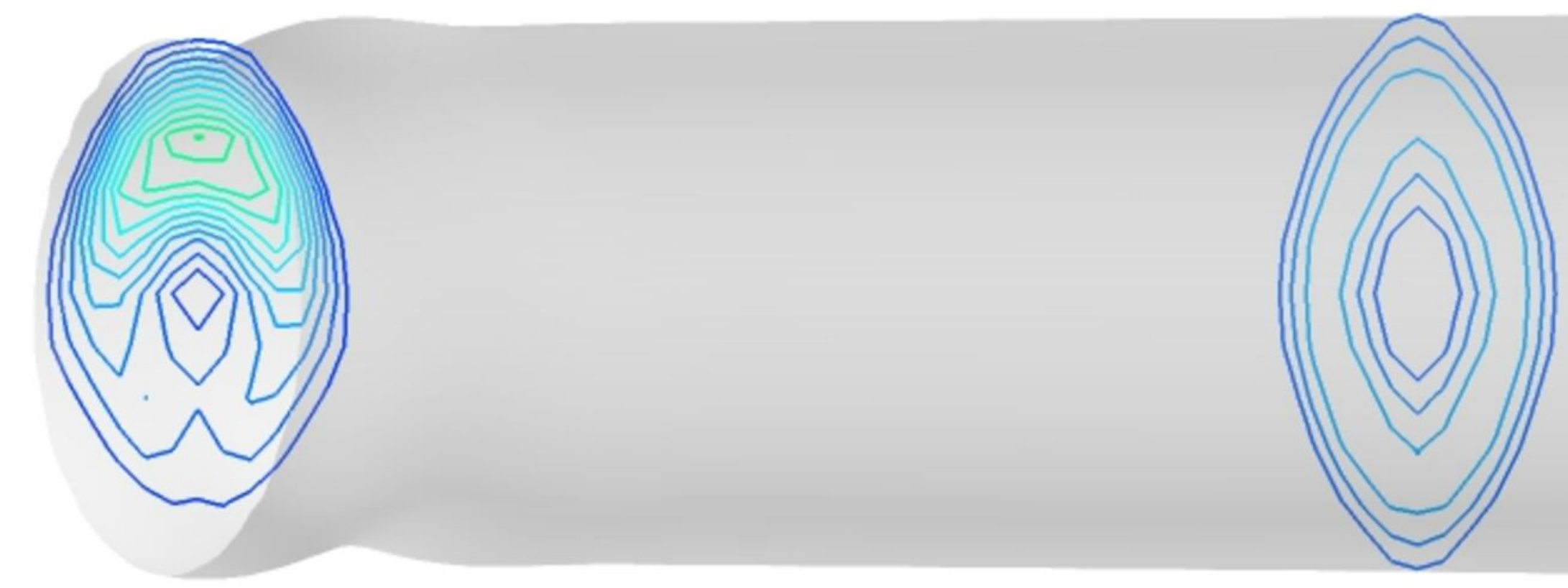
Vorticity Contours on Planes Normal to X-Axis (a) Re=100; Angle=60; Ratio=1:1 (DF)

$1.00 \mathrm{E}+001$ $6.67 \mathrm{E}+000$ $3.33 \mathrm{E}+000$

$0.00 \mathrm{E}+000$

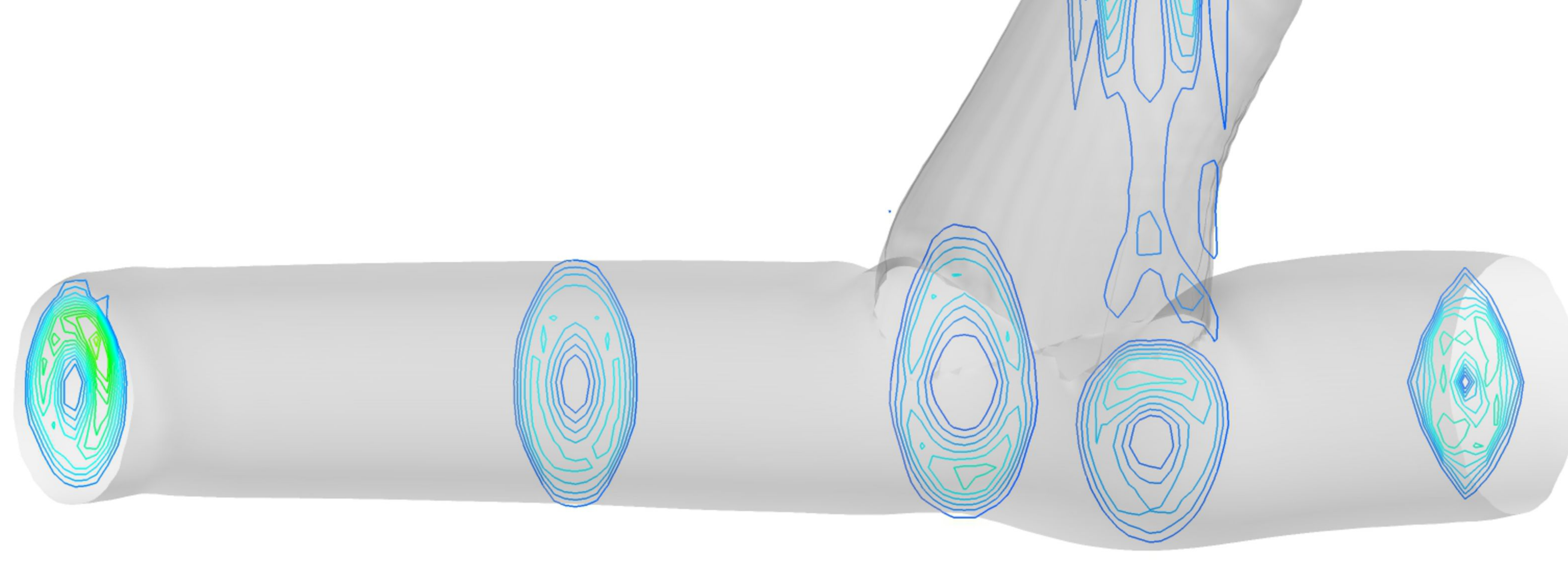




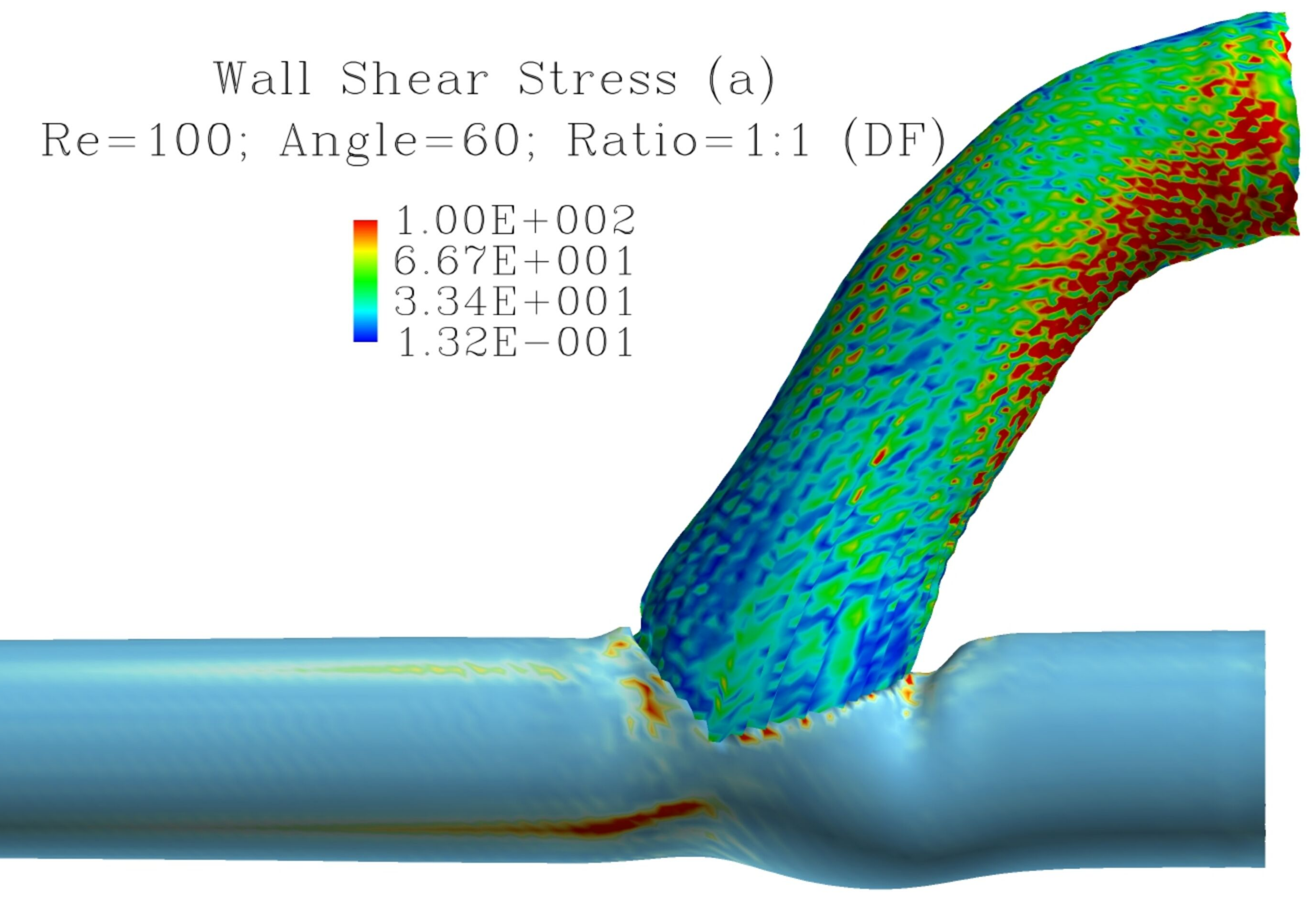

2.00E+001 1.33 $\mathrm{E}+001$ $6.67 \mathrm{E}+000$ $0.00 \mathrm{E}+000$ 
Wall Shear Stress (a)

$$
\mathrm{Re}=100 ; \text { Angle=60; Ratio=1:1 (DF) }
$$

- $1.00 \mathrm{E}+002$ $6.68 \mathrm{E}+001$ $3.36 \mathrm{E}+001$

-3.31E-001

$2.00 \mathrm{E}+001$
$1.33 \mathrm{E}+001$
$6.67 \mathrm{E}+000$
$0.00 \mathrm{E}+000$

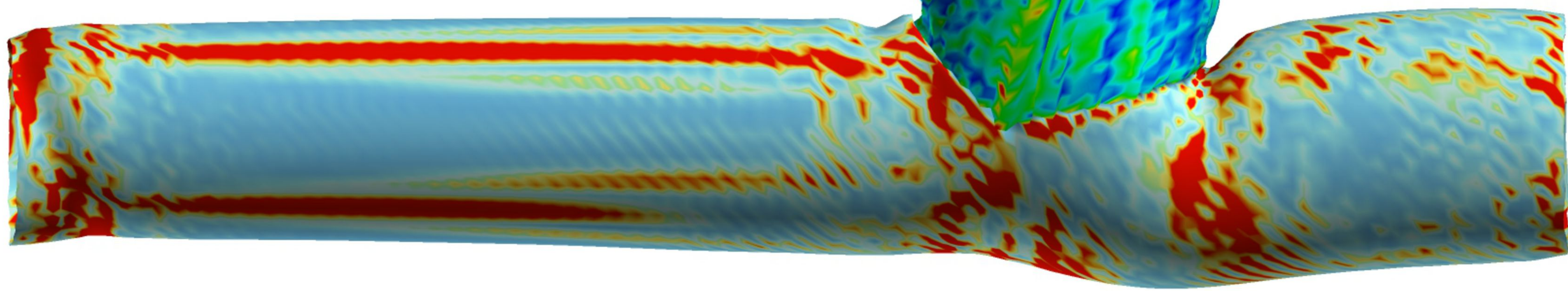




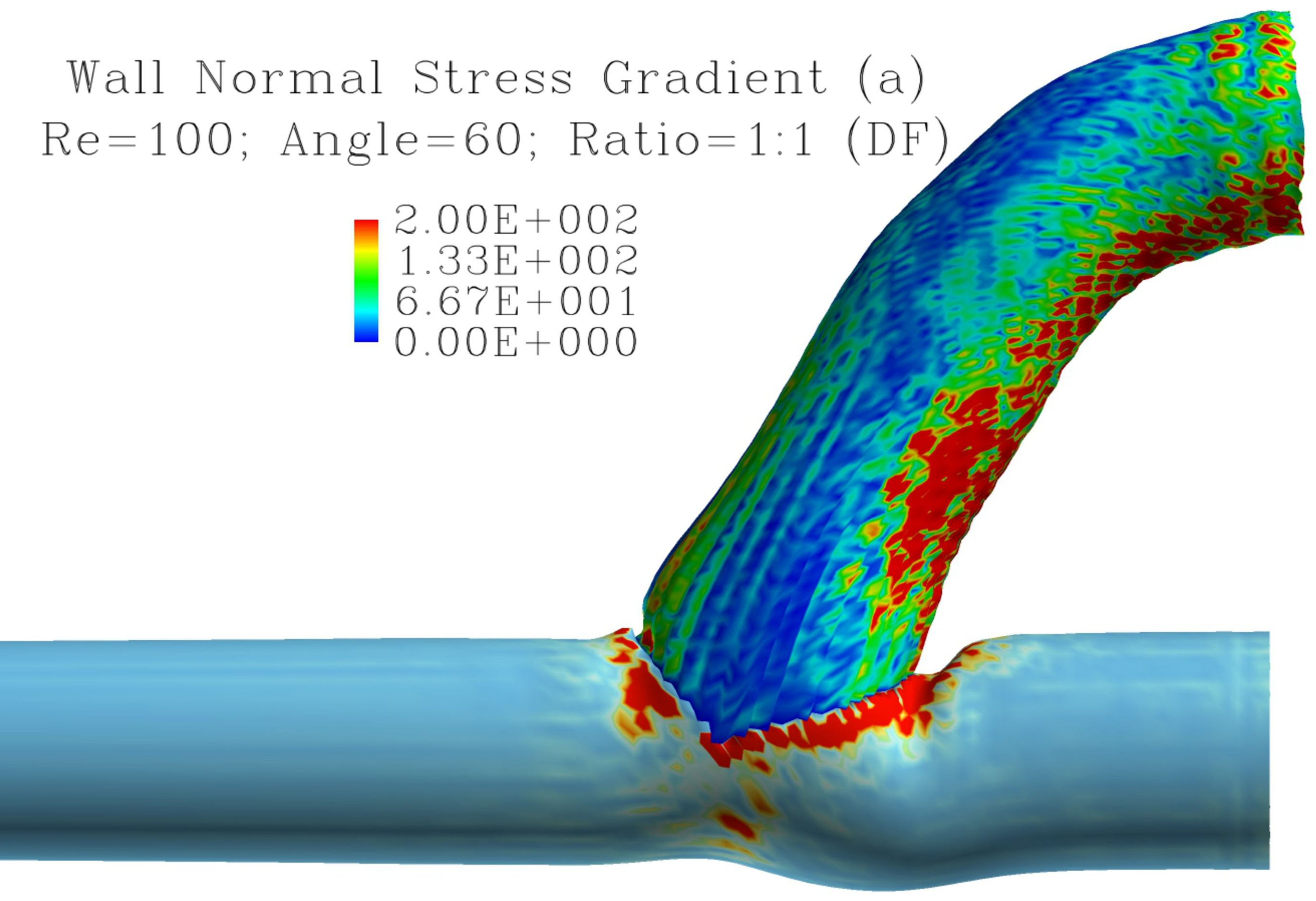

-2.00E+001 1. $33 \mathrm{E}+001$ $6.67 \mathrm{E}+000$ $0.00 \mathrm{E}+000$ 
Wall Normal Stress Gradient (a) Re=100; Angle=60; Ratio=1:1 (DF)

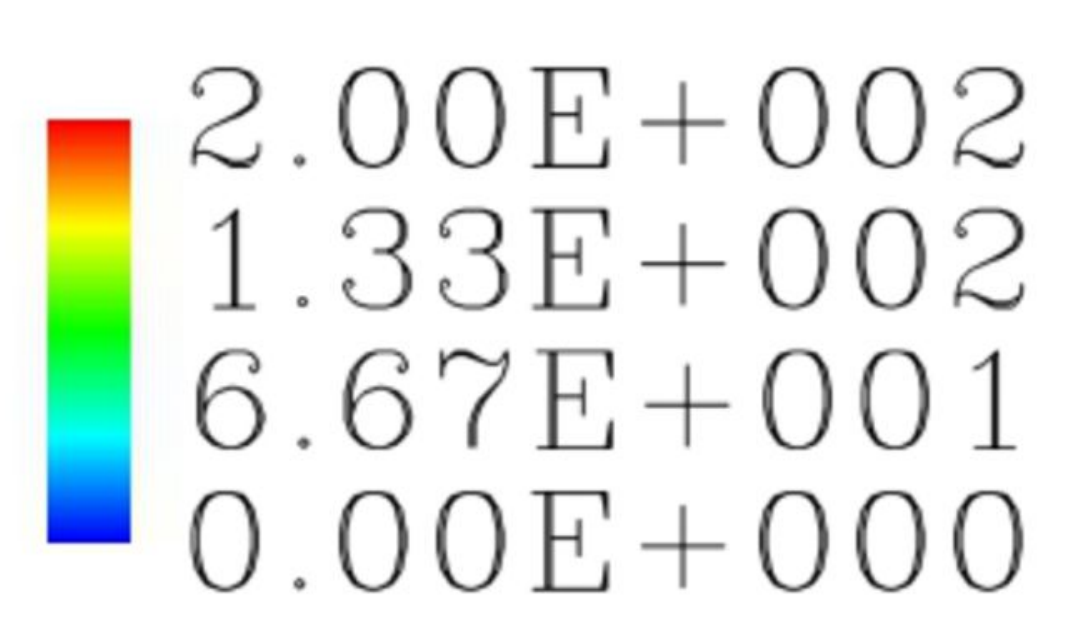

-2.00E+001

1.33E +001

$6.67 \mathrm{E}+000$

$0.00 \mathrm{E}+000$

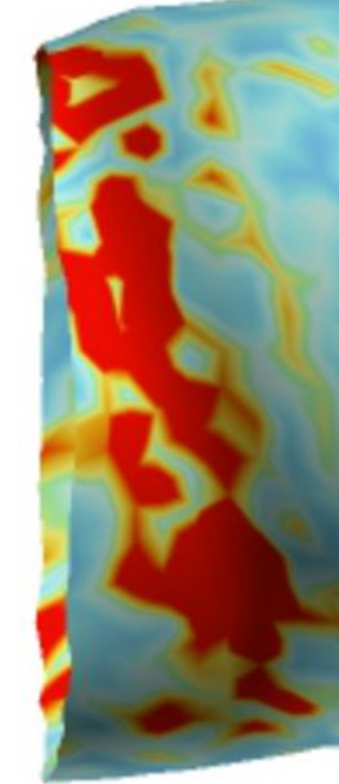

